Archive for

Organic Chemistry

Arkivoc 2017, part iii, 87-104

\title{
Preparation of pyridine-stretched 2'-deoxyhypoxanthosine phosphoramidite
}

\author{
Russell Clayton, ${ }^{\text {a Michael L. Davis, }}$, Wei Li, ${ }^{b}$ William Fraser, ${ }^{* b}$ and Christopher A. Ramsden*a \\ aLennard-Jones Laboratories, School of Physical and Geographical Sciences, Keele University, Keele, \\ Staffordshire, ST5 5BG, UK \\ ${ }^{b}$ School of Life and Health Sciences, Aston University, Aston Triangle, Birmingham, B4 7ET, UK \\ Email:w.fraser@aston.ac.uk, c.a.ramsden@keele.ac.uk
}

\section{Dedicated to Prof. Oleg A. Rakitin on the occasion of his $65^{\text {th }}$ birthday}

Received 02-28-2017

Accepted 03-24-2017

Published on line $\quad 04-10-2017$

\section{Abstract}

Pyridine-stretched 2'-deoxyhypoxanthosine (strH) phosphoramidite was prepared in eight steps from Hoffer's sugar (2'-deoxy-3,5-di-O-(p-toluoyl)- $\alpha$-D-erythro-pentofuranosyl chloride). Improved synthesis of the Hoffer sugar was achieved without need for distillation or chromatographic separation of intermediates, or use of gaseous $\mathrm{HCl}$. Conditions were optimised to provide a key nitrile intermediate for the preparation of strH whereby the cesium salt of 4(5)-nitroimidazole was glycosylated using Hoffer's sugar. The nitrile intermediate was also used to prepare pyridine-stretched 2'-deoxyadenosine (strA) and pyridine-stretched 2'-deoxy-2,6diaminonebulamine (strD). Preliminary studies indicate that strH forms a stronger, size-expanded base pair with adenine compared with the Watson-Crick thymine-adenine base pair.

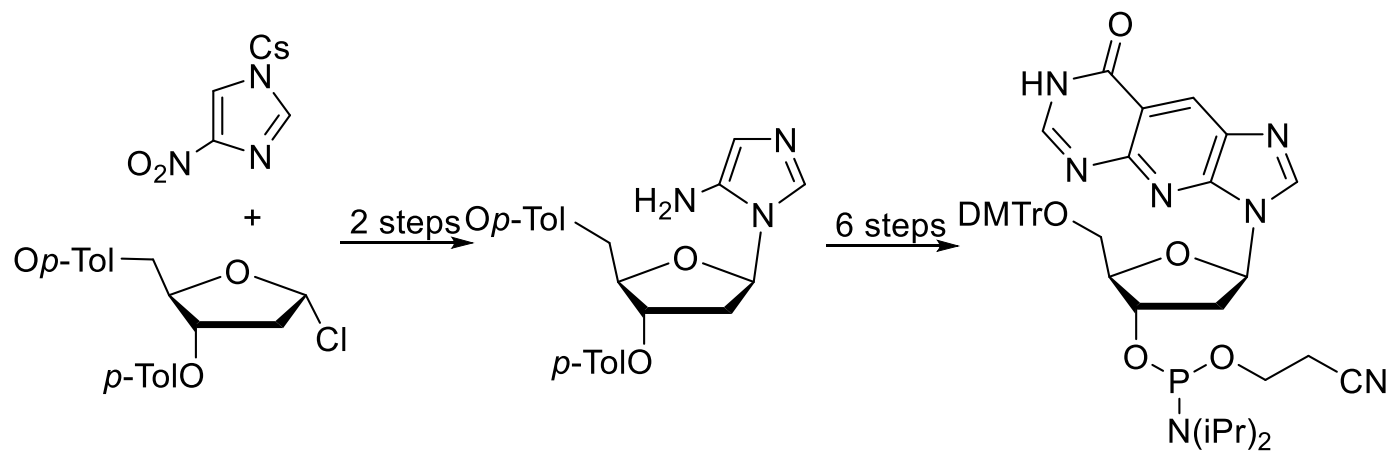

Keywords: 5-Aminoimidazoles, Hoffer's sugar, pyridine-stretched purines, hypoxanthosine, phosphoramidite 


\section{Introduction}

The insertion of a third ring between the pyrimidine and imidazole fragments of purine nucleosides has been investigated by several groups. Leonard and co-workers described the earliest synthesis of the benzenestretched adenosine analogue $1{ }^{1-4}$ Later, the $2^{\prime}$-deoxy derivative $\mathbf{3}$ was prepared (Figure 1). ${ }^{5}$ Recently, benzo homologation of various nucleosides and their oligomerisation has culminated in the realisation of complete series' of benzene-stretched $x D N A^{6-8}$ and $y D N A^{9}$ analogues that are orthogonal to natural DNA. The limits to size expansion have continued to be explored through the study of base-pairs formed between purines and their naphtho-homologated complements in "double-wide DNA" (yyDNA). ${ }^{10}$ "There remains much to be discovered with such size-expanded genetic sets" according to Kool and co-workers. ${ }^{11}$ Size-expanded bases are recognised correctly and with efficiency when inserted into $E$. coli ${ }^{12}$ and can encode amino acids of a protein in a living organism. ${ }^{13}$ Pyridine-stretched adenine is fluorescent and when incorporated into oligomers as the acyclonucleoside 6 provides a useful probe for the detection of single nucleotide polymorphisms in representative DNA and RNA sequences. ${ }^{14-16}$

\section{Results and Discussion}

It has been our aim to assess the effect that pyrido homologation has on the base-pairing and molecular recognition properties of size-expanded oligonucleotides. We have previously described the preparation of adenosine analogue $\mathbf{2}$ and the corresponding xanthosine and hypoxanthosine analogues. ${ }^{17}$ Subsequently, we prepared the $2^{\prime}$-deoxynucleoside analogues of adenine $\mathbf{4}(\operatorname{strA})$ and hypoxanthine $\mathbf{5}(\mathrm{strH}) .{ }^{18}$ More recently we described the synthesis of various 9-substituted adenine and hypoxanthine derivatives. ${ }^{19} \mathrm{~A}$ key intermediate for the synthesis of strH $\mathbf{5}$ and related derivatives is the nitrile $\mathbf{2 4}$ (Scheme 3). Here we describe the preparation of intermediate $\mathbf{2 4}$ and its conversion to the strH nucleoside phorphoramidite $\mathbf{3 0}$ for incorporation into oligonucleotides, together with a preliminary study of the base-pairing properties of strH.
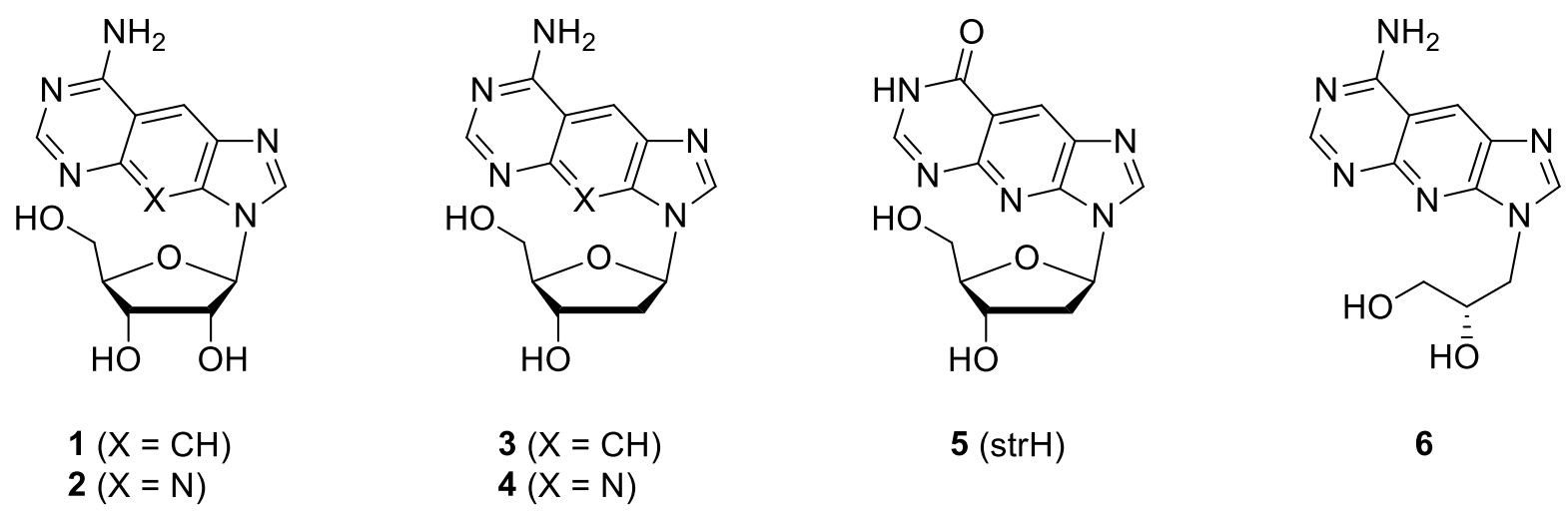

Figure 1. The structures of benzene- and pyridine-stretched nucleosides $\mathbf{1}$ to $\mathbf{5}$ and acyclonucleoside $\mathbf{6}$.

As the starting point for synthesis of the nucleoside intermediate 15 , we selected the well-known and widely used Hoffer sugar (2-deoxy-3,5-di-O-( $p$-toluoyl)- $\alpha$-D-erythro-pentofuranosyl chloride) $10 .{ }^{20}$ It is prepared as a single anomer in three steps from 2 -deoxy- $\alpha / \beta$-D-erythro-pentopyranose $\mathbf{7}$ (Scheme 1 ). A typical Hoffer procedure ${ }^{21}$ gives the sugar 10 as a solid that must then be glycosylated otherwise decomposition 
occurs on storage beyond two weeks, even under vacuum. Improvements to the original procedure have been made. ${ }^{22-24}$ We now describe an improved procedure that increases the yield of the sugar 10 and also circumvents the use of gaseous $\mathrm{HCl}$ or the need for distillation or chromatographic purification of intermediates.

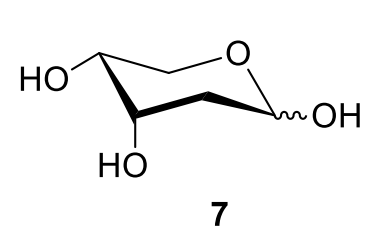

7
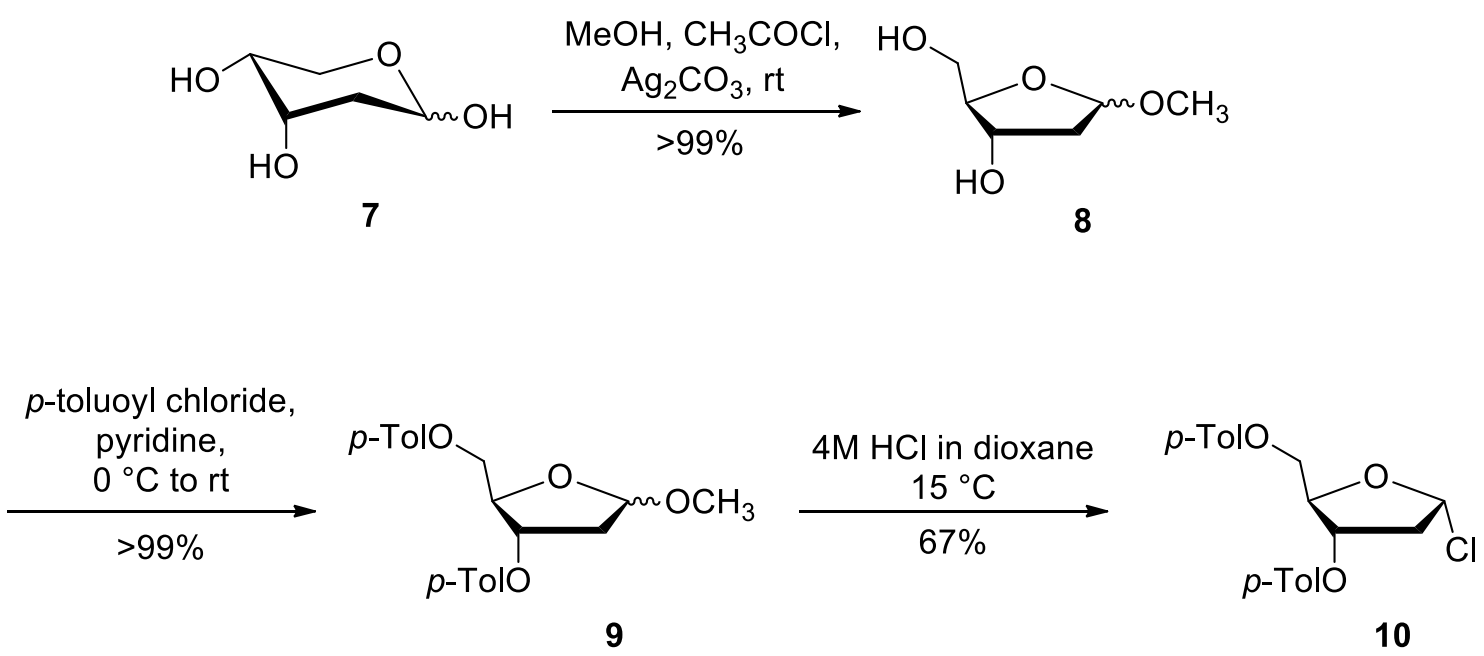

Scheme 1. The preparation of Hoffer's sugar 10 from 2-deoxy- $\alpha / \beta$-D-erythro-pentopyranose 7.

Thus, acetalation of 2-deoxy- $\alpha / \beta$-D-erythro-pentopyranose 7 using methanolic $\mathrm{HCl}$, generated in situ by reaction of acetyl chloride with methanol, gave 1-O-methyl-2-deoxy- $\alpha / \beta$-D-erythro-pentofuranose 8 in quantitative yield. Esterification of $\mathbf{8}$ using $p$-toluoyl chloride in pyridine gave $\mathbf{9}$ as a syrup that, with careful drying, solidified to give $\mathbf{9}$, again in quantitative yield. Chlorination of $\mathbf{9}$ gave the Hoffer sugar $\mathbf{1 0}$ as a white solid (67\%) using $4 \mathrm{M} \mathrm{HCl}$ in dioxane. Samples of sugar 10 prepared by this procedure could be stored without discoloration or deterioration.

In previous glycosylations to prepare ribonucleosides, we used the silver salt of the 4(5)-nitroimidazole anion to give a pair of $\beta$-anomers, where the required $5 \beta$-regioisomer predominated. ${ }^{17}$ We employed the same conditions to carry out glycosylation of the silver salt $\mathbf{1 1}$ using the Hoffer sugar $\mathbf{1 0}$ in xylene to yield a pair of $\beta$-anomers; the required 5-regioisomer 15 was obtained together with the 4-regioisomer 16 in 1:2 ratio (Scheme 2). The required isomer 15 was isolated by flash column chromatography in pure crystalline form. The outcome of glycosylations using $\mathbf{1 0}$ is strongly influenced by choice of solvent and nucleophile. When acetonitrile was used instead of xylene, glycosylation gave an almost even distribution of all four possible isomers 15, 16, 17 and 18.

Use of the lithium salt 12 under these reaction conditions resulted in an increased yield of the $\alpha$ anomers 17 and 18 at the expense of lowered yields of the $\beta$-anomers 15 and $\mathbf{1 6}$. Using the sodium salt 13, generated in situ using sodium hydride, before addition of 10, Bergstrom and co-workers reported isolation of $5 \beta 15$ and $4 \beta 16$ isomers in a ratio of $1: 2 .{ }^{25}$

When we used the preformed sodium salt 13, glycosylation gave a slight improvement in yield and ratio of $\beta$-anomers $15 / 16$ (1:1.5), accompanied by small amounts of the $\alpha$-anomers 17 and 18 . 


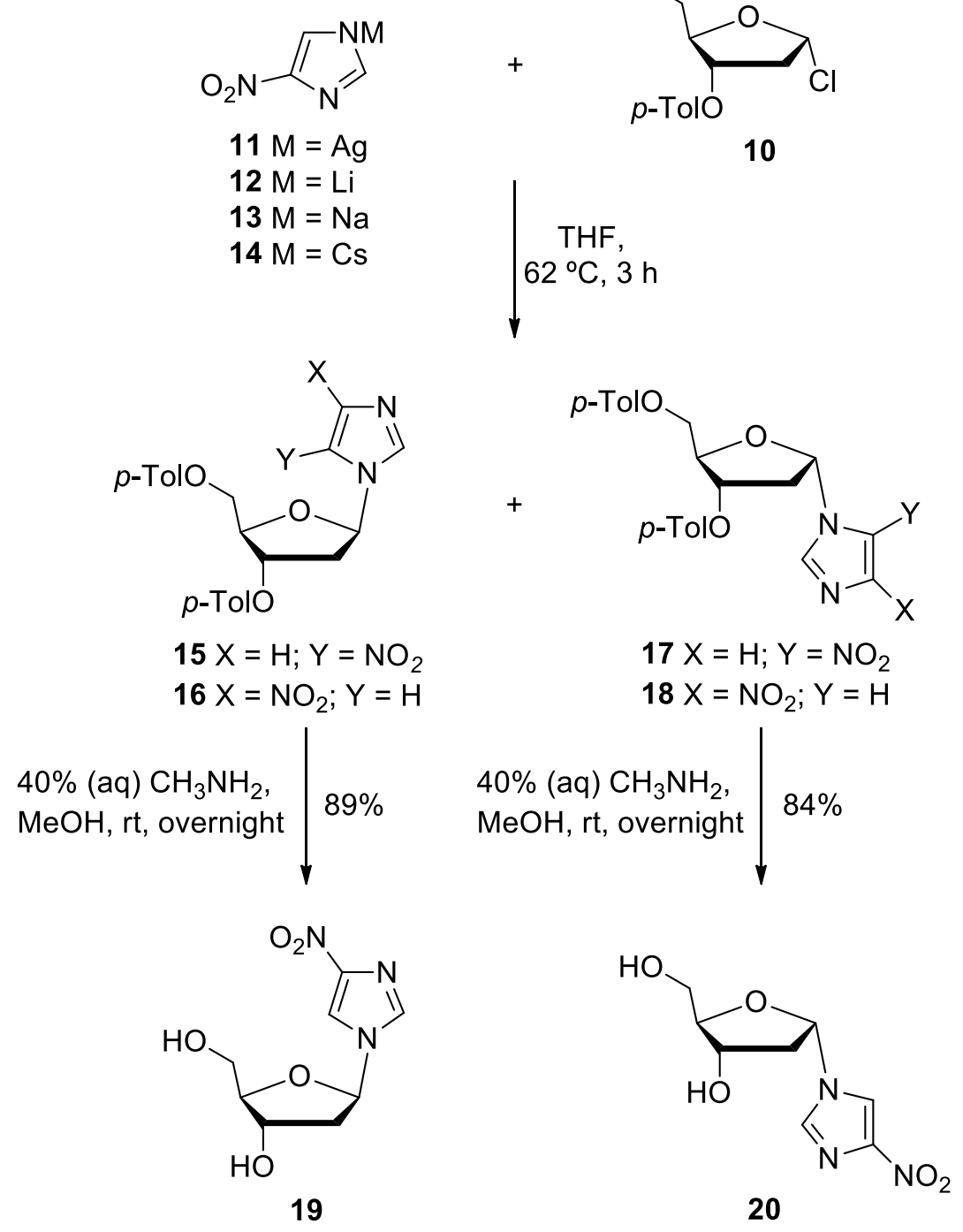

Scheme 2. The glycosylation of Hoffer's sugar 10 using the metal salts 11 to 14 of 4(5)-nitroimidazole.

In the absence of an acyloxy substituent at the $\mathrm{C}^{\prime}$ position, glycosylation is most likely to proceed by an $S_{N} 2$ Walden inversion process to give the $\beta$-configured products. Formation of $\alpha$-configured nucleosides by a similar process can be explained if the sugar $\mathbf{1 0}$ first isomerises. The anomerisation of the $\alpha$-configured sugar 10 to the more reactive $\beta$-anomer is known to occur readily in solvents of higher dielectric constant, and is further enhanced by the presence of lithium, silver and to a lesser extent, sodium ions. ${ }^{26,27}$ During the timescale used for these glycosylation reactions ( $3 \mathrm{~h}$ ), we observed by ${ }^{1} \mathrm{H} N \mathrm{NM}$, that the $\alpha$-configured sugar 10 underwent $40 \%$ inversion to the more reactive $\beta$-anomer in acetonitrile- $d_{3}$ whereas less than $5 \%$ inversion occurred in THF- $d_{8}$. We expected that use of less polar THF for the glycosylation would favour formation of the $\beta$-anomers 15 and 16 over the $\alpha$-anomers 17 and 18 . We reasoned that use of the cesium salt 14 might also lead to an improvement in the anomeric ratio $(\beta / \alpha)$ as $\mathrm{Cs}^{+}$is less likely to co-ordinate to the substituent at $\mathrm{C} 1^{\prime}$ and enhance anomerisation of the $\alpha$-cconfigured sugar 10. Glysosylation of cesium salt 15 with sugar 10 for 3 $\mathrm{h}$ at $62{ }^{\circ} \mathrm{C}$ in THF gave the pure 5-nitro-1'- $\beta$-isomer 15 in an isolated yield of $68 \%$. Clearly, the use of the cesium salt 14 not only improves the anomeric ratio but also improves the regioselectivity 15/16 of the reaction. 


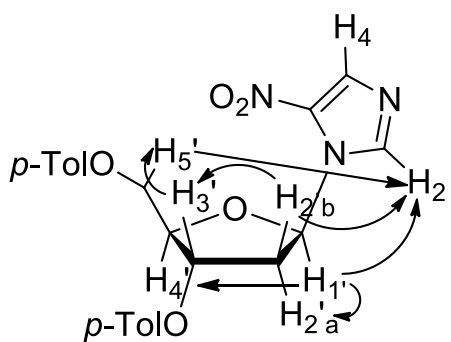

15

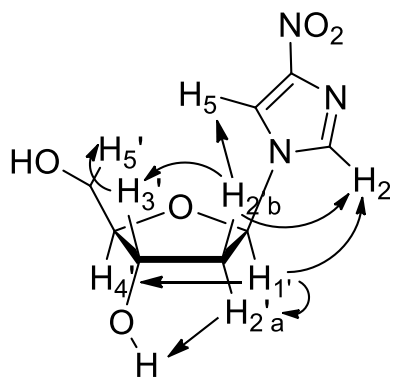

19

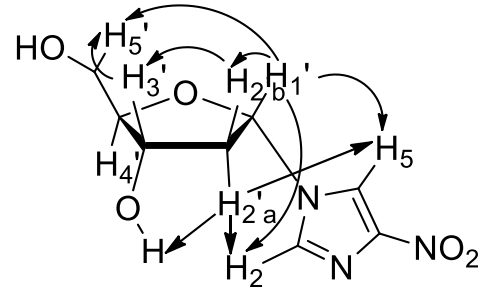

20

Figure 2. Selected NOESY correlations for 5-nitro-1'- $\beta$-isomer 15, 4-nitro-1'- $\beta$-isomer 19 and 4-nitro-1'- $\alpha$ isomer 20.

The identities of all four isomers 15 to 18 were established by ${ }^{1} \mathrm{H}$ and ${ }^{13} \mathrm{C}$ NMR analysis with 5-nitro-1'- $\beta$ 15 (triplet, $\delta 6.72 \mathrm{ppm}$ ) and 5-nitro-1'- $\alpha$ (doublet, $\delta 6.80 \mathrm{ppm}) 17$ anomeric proton signals being downfield relative to those of the 4-nitro-1'- $\beta 16$ (triplet, $\delta 6.17$ ppm) and 4-nitro-1'- $\alpha 18$ (doublet, $\delta 6.46$ ppm) signals. The positions of carbon signals of 5-nitro-1'- $\beta$-isomer 15 (C2 and C4 = 138.13 and 134.33 ppm; C5 not observed) and 4-nitro-1'- $\beta$-isomer 16 (C2, C4 and $C 5=134.28,148.49$ and 116.79 ppm) were in agreement with the respective chemical shifts reported for 1-substituted-5- and 1-substituted-4-nitroimidazoles. ${ }^{17,28}$

The identities of compound 15 and compounds 19 and 20, which are the deprotected derivatives of isomers 16 and 18, were further confirmed by NOESY correlations (Figure 2). Removal of the $p$-toluoyl protecting groups from compounds 16 and 18 was achieved using methylamine in methanol to give nucleosides 19 and 20 . Under the same conditions, attempts at deprotection of the 5-nitro-1'- $\beta$-isomer $\mathbf{1 5}$ failed. The 5-nitro-1'- $\alpha$-isomer 17 was unavailable in sufficient quantity to attempt deprotection. The 5nitroimidazole nucleoside $\mathbf{1 5}$ was catalytically reduced to give amine $\mathbf{2 1}$ that was promptly reacted with ethoxymethylene malononitrile (Scheme 3).

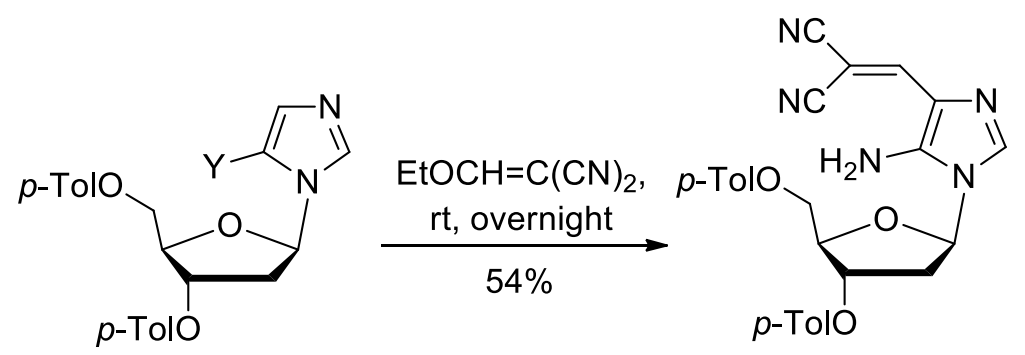

22

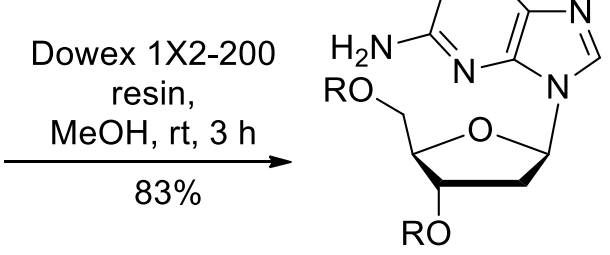

$23 \mathrm{R}=p-\mathrm{Tol}$

$24 \mathrm{R}=\mathrm{H}$

Scheme 3. The preparation of the key nitrile nucleoside intermediate 24.

Although amine 21 could be characterised by proton NMR analysis ( $\mathrm{NH}_{2}$, broad singlet, $\delta 4.01 \mathrm{ppm}$ ) it was prone to decomposition. However, it proved convenient to generate the amine $\mathbf{2 1}$ from compound $\mathbf{1 5}$ for use directly in the next step, when required. Use of ethoxymethylene malononitrile as electrophile gave exclusively the C-addition-elimination product $\mathbf{2 2}$ in accord with our previous work with 5- 
aminoimidazoles. ${ }^{19,29,30}$ Compound 22 showed a primary amine signal ( $\delta 7.72 \mathrm{ppm}$ ) and a single imidazole proton ( $\delta 7.83 \mathrm{ppm})$.

Cyclisation of compound $\mathbf{2 2}$ was achieved by stirring with ion exchange resin to give the protected nitrile nucleoside 23. Although the pure, protected nitrile nucleoside $\mathbf{2 3}$ was isolated and characterised, it was possible to carry out cyclisation of compound $\mathbf{2 2}$ and deprotection concomitantly, to give the key nitrile nucleoside intermediate 24. This product (24), formed in two isolation steps from the 4(5)-nitroimidazole cesium salt 14 (Scheme 2), is a key common intermediate for the preparation of other size-expanded nucleosides $\mathbf{4}$ and $\mathbf{3 1}$ (Scheme 6).

Direct glycosylation of the sodium salt of 5-aminoimidazo[4,5-b]pyridine-6-carbonitrile (AIPC) $25^{31}$ using Hoffer's sugar 10 provided a convergent route to the protected nitrile nucleoside 23 (Scheme 4). The sodium salt of compound $\mathbf{2 5}$ was generated in situ and a solution (THF) of the sugar 10 was added dropwise $(15 \mathrm{~min})$ at $55^{\circ} \mathrm{C}(2 \mathrm{~h})$ to give a $1: 2$ mixture of the $\beta$-anomers 23 and $\mathbf{2 6}$. The pure compounds $\mathbf{2 3}(19 \%)$ and 26 $(22 \%)$ were readily isolated after separation by flash column chromatography. When the same reaction was carried out but with the sugar added immediately as a single portion at room temperature ( $2 \mathrm{~h})$, the anomeric ratio improved giving a near 1:1 mixture of $\beta$-anomers $\mathbf{2 3}$ and $\mathbf{2 6}$ but in lower overall yield. In this case, pure compound 23 (14\%) was readily isolated.

Although the direct glycosylation of Hoffer's sugar using AIPC 25 has not been optimised, our preferred route to the key nitrile intermediate is the longer one via nitroimidazole nucleoside 15 . AIPC 25 is readily prepared in high yield $(90 \%)^{31}$ by a straightforward reaction between purine with malononitrile. However, purine is expensive to source commercially. The simplest and most direct of the various synthetic routes to purine involves heating neat formamide $\left(>160^{\circ} \mathrm{C}\right)$ but the isolated yield $(14.4 \%)$ of pure product is very modest. $^{32,33}$ It was possible to recover unreacted formamide and subject it to further reaction without deterioration in the yield of purine.

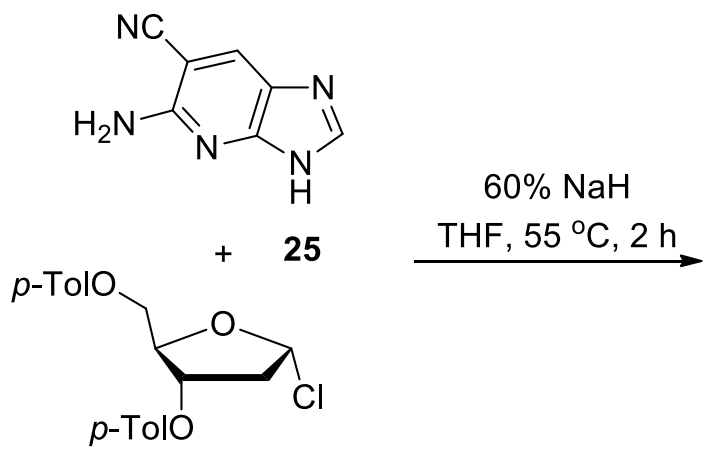

10

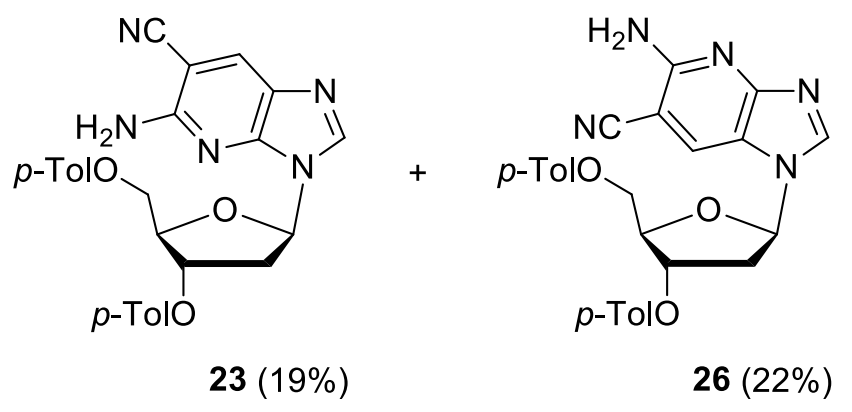

Scheme 4. The preparation of protected nitrile $\mathbf{2 3}$ by direct glycosylation of the sodium salt of compound $\mathbf{2 5}$ using Hoffer's sugar 10.

The preparation of amide 27 from nitrile 24 was achieved in $79 \%$ yield using $\mathrm{H}_{2} \mathrm{O}_{2}$ and aq $\mathrm{NH}_{3}$ or directly from amine 22 in $67 \%$ yield by refluxing $(3 \mathrm{~h}$ ) with $\mathrm{NaOH}$ in $\mathrm{MeOH}$ (Scheme 5). Subsequent cyclisation of the amide $\mathbf{2 7}$ using ethyl formate and sodium ethoxide under reflux ( 2 h) gave strH nucleoside $\mathbf{5}$ in $94 \%$ yield. 


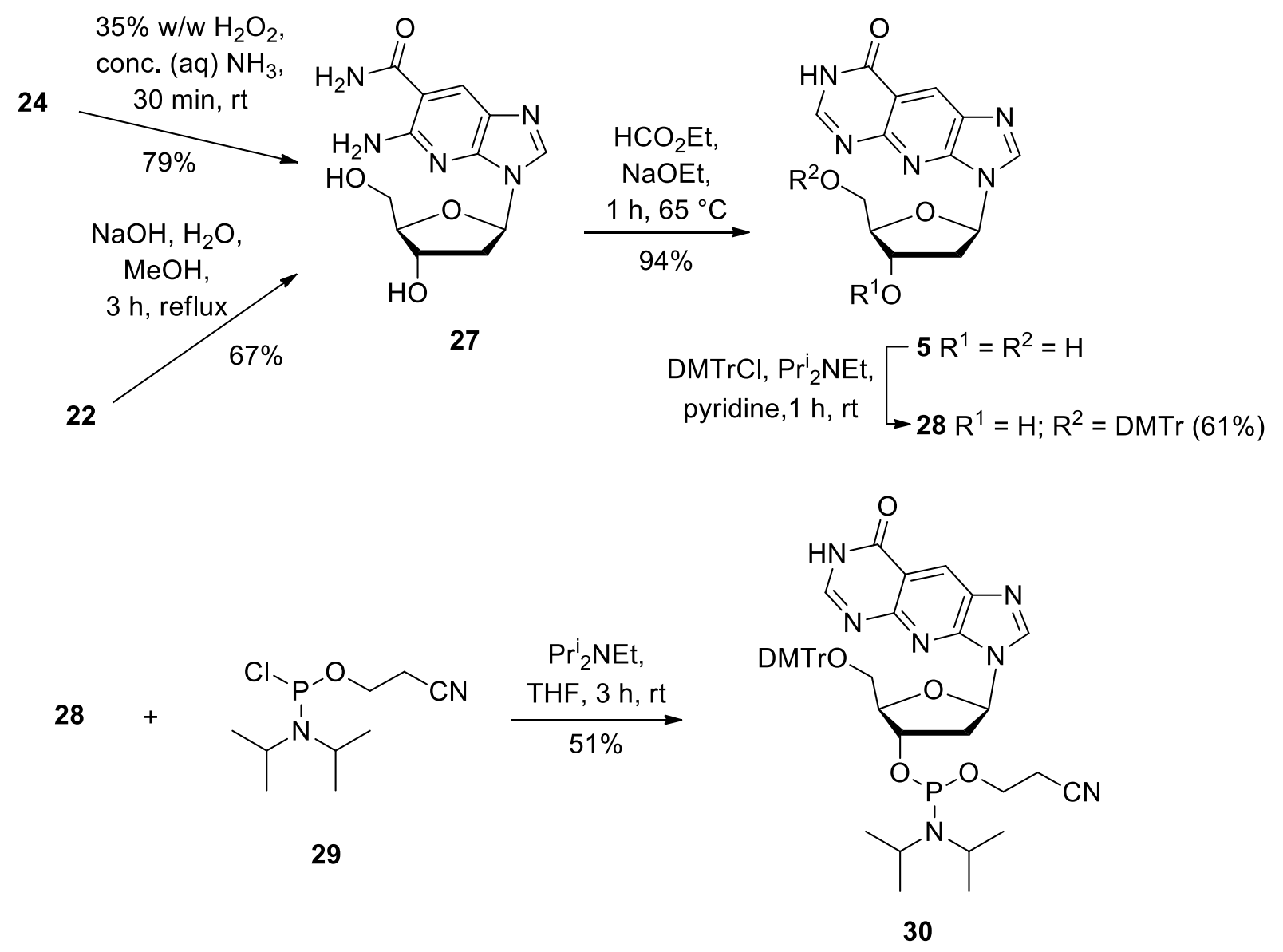

Scheme 5. The preparation of strH phosphoramidite $\mathbf{3 0}$ from key nitrile nucleoside intermediate 24.

Nucleoside phosphoramidites containing hypoxanthine have been incorporated into oligonucleotides by automated solid phase synthesis without the need for additional protection of the carbonyl. ${ }^{34,35}$ We therefore anticipated that no protection of the carbonyl in strH $\mathbf{5}$ would be required. Dimethoxytritylation of strH 5 provided the O5'-protected derivative 28 (61\%) that underwent phosphitylation at O3' using compound 29 and standard conditions to give the strH nucleoside phosphoramidite $\mathbf{3 0}$ (51\%).

Preparation of the key nitrile intermediate $\mathbf{2 4}$ also provides the opportunity to make other sizeexpanded nucleosides (Scheme 6). Thus, compound $\mathbf{2 4}$ in hot diethoxymethyl acetate gave the $O$ acetylimidate which, upon treatment with methanolic ammonia, gave the formamidine. Subsequent treatment with aqueous acetic acid and neutralization gave crystalline strA 4 (47\%).

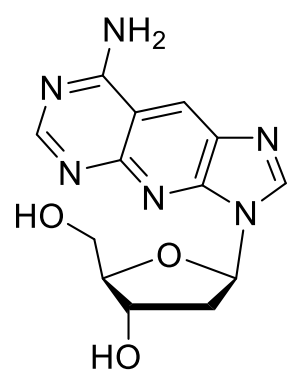

$4(\operatorname{str} A)$
1. $\mathrm{MeCO}_{2} \mathrm{CH}(\mathrm{OEt})_{2}$,

reflux, $2 \mathrm{~h}$

2. $\mathrm{MeOH}, \mathrm{NH}_{3}$

$47 \%$

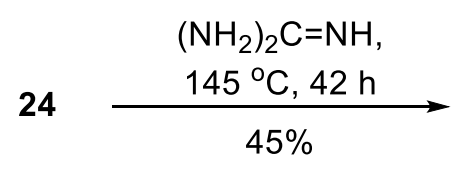

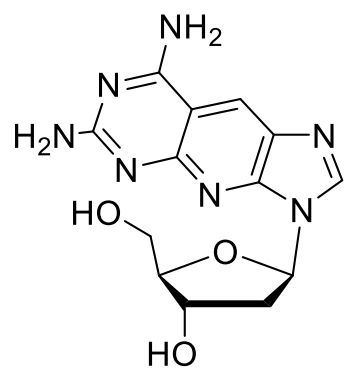

$31(\operatorname{strD})$

Scheme 6. The preparation of pyridine-stretched 2'-deoxy nucleosides 4 (strA) and 31 (strD). 
The nitrile nucleoside 24 was converted to strD 31 (45\%) in a single step by reaction with guanidine in a steel bomb at $145^{\circ} \mathrm{C}$. The structures of strA 4 and strD 31 were fully supported by spectroscopic and analytical data.

Preliminary investigation showed strH nucleoside phosphoramidite $\mathbf{3 0}$ to be compatible with standard, automated solid-supported oligonucleotide synthesis protocols. Variable temperature UV measurements were used to show that replacement strH was capable of forming Watson-Crick base pairs with adenine (A) to give size-expanded structures formed from a purine and tricyclic pyrimidine-stretched purine (Figure 3). When each thymine $(T)$ base in self-complementary DNA oligomer $5^{\prime}$-dATAATATTAT $32\left(T_{m} 28.4 \pm 0.5{ }^{\circ} \mathrm{C}\right)$ was replaced by strH $(\underline{H})$, a significant increase in duplex stability was observed for $5^{\prime}$-dA $\underline{\text { HAA }} \underline{\mathrm{H}} \underline{\mathrm{H}} \underline{\mathrm{H}} \mathrm{A} \underline{\mathrm{H}} 33\left(T_{\mathrm{m}} 42.7 \pm\right.$ $0.5^{\circ} \mathrm{C}$ ) equivalent to $+2.9^{\circ} \mathrm{C}$ per replacement.

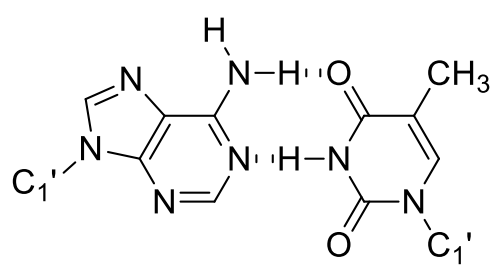

A

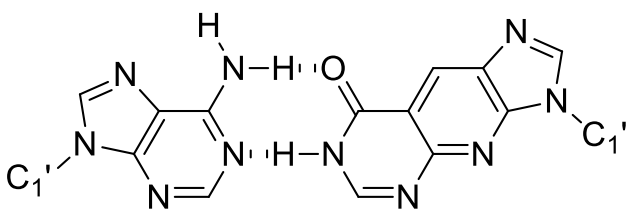

A

strH

Figure 3. The Watson-Crick A.T base pair and size-expanded A.strH base pair where $\mathrm{C}_{1^{\prime}}$ represents the position of attachment to the sugar-phosphate backbone in DNA oligomers $\mathbf{3 2}$ and $\mathbf{3 3 .}$

\section{Conclusions}

We have established two routes to the key nitrile nucleoside $\mathbf{2 4}$ intermediate that allows access to strH 5, strA 4 and strD 31; the pyridine-stretched analogues of 2'-deoxy-hypoxanthosine, 2'-deoxy-adenosine and 2,6diaminonebulamine. The slightly longer route via nitroimidazole nucleoside $\mathbf{2 4}$ is preferred as the more convergent route to the protected key nitrile nucleoside $\mathbf{2 4}$ relies on AIPC $\mathbf{2 5}$ sourced from purine, which is itself expensive and time-consuming to synthesise in suitable quantities. Nucleoside $\mathbf{2 3}$ was converted to the phosphoramidite $\mathbf{3 0}$ which was found to be compatible with standard, solid-supported methods of automated synthesis. We demonstrated formation of size-expanded A.strH base pairing that is more stable by $+2.9{ }^{\circ} \mathrm{C}$ per replacement compared with A.T Watson-Crick base pairing.

\section{Experimental Section}

General. NMR spectra were recorded on Bruker AC-250, Bruker Avance 400 or Jeol 400 spectrometers with ${ }^{1} \mathrm{H}$ spectra referenced to TMS, ${ }^{13} \mathrm{C}$ spectra referenced to $\mathrm{CDCl}_{3}$ or $\mathrm{DMSO}-d_{6}$, and ${ }^{31} \mathrm{P}$ spectra referenced to $85 \%$ $\mathrm{H}_{3} \mathrm{PO}_{4}(\mathrm{aq})$. Mass spectra were recorded in chemical ionisation (CI) or electrospray ionisation (ES) mode with a Hewlett-Packard HP 5989B MS Engine apparatus using a HP 59987A API-electrospray LC/MS interface. Electron impact ionisation (EI) mass spectra (70eV) were recorded on a AEI MS1Z mass spectrometer. Infrared spectra were recorded using a Mattson Galaxy 2020 FT-IR Spectrophotometer. Ultraviolet spectra were recorded using a Unicam PU8730 Spectrophotometer. Melting points were measured on a Gallenkamp Electrothermal Digital apparatus and are uncorrected. Flash column chromatography was performed using Sorbsil C60 silica using the 
method described by Still, Kahn and Mitra. ${ }^{36}$ Dry column vacuum chromatography was performed with Merck Silica gel $60(15-40 \mu \mathrm{m})$ using the method described by Pedersen and Rosenbohm. ${ }^{37}$ TLC was carried out on pre-coated Merck 60 F254 aluminium-backed plates and visualized using UV (254 and $360 \mathrm{~nm}$ ) and vanillin reagent; vanillin $(6.0 \mathrm{~g})$, ethanol $(250 \mathrm{~mL})$ and conc. sulfuric acid $(2 \mathrm{~mL})$. Ethoxymethylene malononitrile (Aldrich) was purified by flash column chromatography eluting with diethyl ether to give a light yellow crystalline solid.

1-O-Methyl-2-deoxy- $\alpha / \beta$-D-erythro-pentofuranose (8). To a stirred solution of 2-deoxy- $\alpha / \beta$-D-erythropentopyranose $7(40.0 \mathrm{~g}, 0.30 \mathrm{~mol})$ in methanol $(600 \mathrm{~mL})$ a solution of acetyl chloride $(1.35 \mathrm{~mL}, 20 \mathrm{~mol}) \mathrm{in}$ methanol $\left(70 \mathrm{~mL}\right.$ ) was added dropwise $(20 \mathrm{~min})$ at $\mathrm{rt}$. After further stirring (15 min), $\mathrm{Ag}_{2} \mathrm{CO}_{3}(13.1 \mathrm{~g}, 47 \mathrm{mmol})$ was added and stirring continued (20 min). The resulting mixture was filtered through acid-washed celite and the filter cake washed with $\mathrm{MeOH}(3 \times 30 \mathrm{~mL})$. The filtrate plus washings were concentrated under reduced pressure and dried under high vacuum to yield compound 8 (44.1 $\mathrm{g},>99 \%)$ as a light brown oil composed of a mixture of $\alpha / \beta$-anomers. $R_{f} 0.40$ (EtOAc-MeOH 4:1). ${ }^{1} \mathrm{H} \mathrm{NMR}\left(250.1 \mathrm{MHz}, \mathrm{CDCl}_{3}\right): \delta 1.63,1.93$ and 2.23 (3 x m, $\left.2 \mathrm{H}, 2-\mathrm{CH}_{2}\right), 3.20\left(\mathrm{~s}, 1.5 \mathrm{H}, 1-\beta-\mathrm{OCH}_{3}\right), 3.22\left(\mathrm{~s}, 1.5 \mathrm{H}, 1-\alpha-\mathrm{OCH}_{3}\right), 3.39,3.48$ and $3.65\left(3 \times \mathrm{m}, 2 \mathrm{H}, 5-\mathrm{CH}_{2}\right), 3.89$ and $4.08(2 \times \mathrm{m}, 1 \mathrm{H}, 3-\mathrm{CH}), 4.63(\mathrm{~m}, 1 \mathrm{H}, 5-\mathrm{OH}), 4.88$ and $4.94(2 \times \mathrm{m}, 1 \mathrm{H}, 1-\mathrm{CH}) 4.83(\mathrm{~d}, J 5.0 \mathrm{~Hz}, 0.5 \mathrm{H}, 3-\mathrm{OH}), 4.98$ (d, J $5.0 \mathrm{~Hz}, 0.5 \mathrm{H}, 3-\mathrm{OH})$.

1-O-Methyl-2-deoxy-3,5-di-O-( $\boldsymbol{0}$-toluoyl)- $\alpha / \beta$-D-erythro-pentofuranose (9). The pentofuranose 8 (44.0 g, 0.3 mol) was co-evaporated twice with pyridine $(2 \times 140 \mathrm{~mL})$, and then dissolved in dry pyridine $(170 \mathrm{~mL})$ while stirring under an argon atmosphere. The solution was then chilled (ice/ $\mathrm{NaCl} /$ water bath) and $p$-toluoyl chloride $(100 \mathrm{~g}, 0.65 \mathrm{~mol})$ was added dropwise $(40 \mathrm{~min})$. The resulting pink slurry was allowed to warm to rt and stirring was continued ( $24 \mathrm{~h}$ ). The reaction mixture was evaporated under reduced pressure and concentrated under high vacuum. The residue was partitioned between ether $(400 \mathrm{~mL})$ and water $(400 \mathrm{~mL})$, and the organic layer was washed with $0.5 \mathrm{M} \mathrm{HCl}(\mathrm{aq})(3 \times 200 \mathrm{~mL})$, saturated $\mathrm{NaHCO}_{3}(\mathrm{aq})(3 \times 200 \mathrm{~mL})$ and $5 \% \mathrm{NaCl}(\mathrm{aq})(3 \times 200$ $\mathrm{mL}$ ). The ether layer was dried (anhyd. $\mathrm{MgSO}_{4}$ ), concentrated under reduced pressure and dried under high vacuum to yield compound $\mathbf{9}$ as a clear, light orange coloured syrup. Further drying under high vacuum on a slowly rotating rotary evaporator caused crystals to slowly form. On further standing the product 9 (114.6 g, $>99 \%$ ) slowly solidified to give a mixture of $\alpha / \beta$-anomers as pale buff crystals. Spectroscopic and analytical data were in agreement with the data published previously. ${ }^{22} R_{f} 0.49$ and 0.52 (EtOAc-Hexane $1: 2$ ). ${ }^{1} \mathrm{H} N M R$ $\left(250.1 \mathrm{MHz}, \mathrm{CDCl}_{3}\right): \delta 2.40\left(\mathrm{~m}, 8 \mathrm{H}, 2-\mathrm{CH}_{2}, 2 \times 4\right.$-tol- $\left.\mathrm{CH}_{3}\right), 3.36\left(\mathrm{~s}, 1.5 \mathrm{H}, 1-\beta-\mathrm{OCH}_{3}\right), 3.43(\mathrm{~s}, 1.5 \mathrm{H}, 1-\alpha-\mathrm{OCH}), 4.45$ $\left(\mathrm{m}, 2 \mathrm{H}, 5-\mathrm{CH}_{2}\right), 5.19(\mathrm{~d}, J 4.9 \mathrm{~Hz}, 0.5 \mathrm{H}, 1-\mathrm{CH \alpha}), 5.21(\mathrm{~m}, 0.5 \mathrm{H}, 4-\mathrm{CH}), 5.40(\mathrm{~m}, 0.5 \mathrm{H}, 4-\mathrm{CH}), 5.61(\mathrm{~m}, 0.5 \mathrm{H}, 1-$ $\mathrm{CH} \beta), 7.23$ (m, 4H, 3,5-tol-CH), 7.97 ( $m, 4 \mathrm{H}, 2,6$-tol- $\mathrm{CH})$.

2-Deoxy-3,5-di-O-( $\boldsymbol{p}$-toluoyl)- $\alpha$-D-erythro-pentofuranosyl chloride (Hoffer's sugar) (10). The pentofuranose 9 $(50.0 \mathrm{~g}, 0.13 \mathrm{~mol})$ was dissolved in glacial acetic acid $(150 \mathrm{~mL})$ by stirring at $\mathrm{rt}$ for 20 min under an argon atmosphere. The solution was cooled to $10-15{ }^{\circ} \mathrm{C}$ and $4 \mathrm{M} \mathrm{HCl}$ in dioxane $(150 \mathrm{~mL}, 0.6$ mol) was added dropwise $(5 \mathrm{~min})$. The pale, straw-coloured solution was seeded with a small amount of compound 10 and stirred briefly until a precipitate started to form. The stirring was stopped and the reaction mixture stood (40 min) with cooling (ice/water). The resultant white slurry was broken up by swirling the flask and filtered rapidly. The filter cake was packed down, washed with cold ether $(3 \times 30 \mathrm{~mL})$ and dried under high vacuum to give compound 10 (34.1 g, 67\%) as a white solid. Spectroscopic and analytical data were in agreement with the data published previously. ${ }^{22} R_{f} 0.58$ (EtOAc-Hexane 1:2). IR $\left(\mathrm{cm}^{-1}\right)$ : 3030, 2951, 1708, 1276, 1178, 1099, $755 .{ }^{1} \mathrm{H}$ NMR (250.1 MHz, CDCl $): \delta 2.41\left(\mathrm{~s}, 3 \mathrm{H}, \mathrm{CH}_{3}\right), 2.42\left(\mathrm{~s}, 3 \mathrm{H}, \mathrm{CH}_{3}\right), 2.74(\mathrm{~m}, 1 \mathrm{H}, 2-\mathrm{CH}), 2.84(\mathrm{~m}, 1 \mathrm{H}, 2-\mathrm{CH}), 4.61(\mathrm{dd}$, J 12.1, $4.1 \mathrm{~Hz}, 1 \mathrm{H}, 5-\mathrm{CH}), 4.66(\mathrm{dd}, J 12.1 \mathrm{~Hz}, 4.1 \mathrm{~Hz}, 1 \mathrm{H}, 5-\mathrm{CH}), 4.85(\mathrm{~m}, 1 \mathrm{H}, 4-\mathrm{CH}), 5.58(\mathrm{~m}, 1 \mathrm{H}, 3-\mathrm{CH}), 6.47(\mathrm{~d}, J$ $4.2 \mathrm{~Hz}, 1 \mathrm{H}, 1-\mathrm{CH}), 7.24$ (d, J $8.3 \mathrm{~Hz}, 2 \mathrm{H}, 3,5$-tol-CH), 7.26 (d, J $8.3 \mathrm{~Hz}, 2 \mathrm{H}, 3,5$-tol-CH), 7.90 (d, J 8.3 Hz, 2H, 2,6- 
tol-CH), 7.99 (d, J $8.3 \mathrm{~Hz}, 2 \mathrm{H}, 2,6$-tol-CH). ${ }^{13} \mathrm{C} \mathrm{NMR}\left(62.9 \mathrm{MHz}, \mathrm{CDCl}_{3}\right): \delta 21.70\left(\mathrm{CH}_{3}\right), 21.71\left(\mathrm{CH}_{3}\right), 44.54\left(2-\mathrm{CH}_{2}\right)$, $63.51\left(5-\mathrm{CH}_{2}\right), 73.56(4-\mathrm{CH}), 84.71(3-\mathrm{CH}), 95.3(1-\mathrm{CH}), 126.66(\mathrm{C}), 126.72(\mathrm{C}), 129.19(\mathrm{CH}), 129.21(\mathrm{CH}), 129.63$ (CH), $129.88(\mathrm{CH}), 144.07$ (C), 144.30 (C), 166.07 (CO), $166.41(\mathrm{CO}) . \mathrm{MS}(\mathrm{EI}): \mathrm{m} / z(\%) 353\left(\mathrm{M}^{-}-\mathrm{Cl}_{1}, 1\right), 252(1)$, 216 (32), 136 (38), 119 (100), 91 (78), 81 (100), 65 (28), 53 (25), 39 (19). Anal. Calcd for $\mathrm{C}_{21} \mathrm{H}_{22} \mathrm{ClO}_{5}: \mathrm{C}, 64.8 ; \mathrm{H}$, 5.41. Found: C, 64.4; $\mathrm{H}, 5.39$.

4(5)-Nitroimidazole silver salt (11). Nitroimidazole $\left(4.52 \mathrm{~g}, 40 \mathrm{mmol}\right.$ ) was dissolved in conc. aq $\mathrm{NH}_{3}(70 \mathrm{~mL})$ to give a clear yellow solution. A solution of silver nitrate $(6.8 \mathrm{~g}, 40 \mathrm{mmol})$ in water $(40 \mathrm{~mL})$ was added dropwise (20 $\mathrm{min}$ ) with moderate stirring at $\mathrm{rt}$. After further stirring $(10 \mathrm{~min})$ the resultant pale yellow precipitate was filtered off, washed well with ethanol and dried under high vacuum to give the product 11 (8.16 g, 93\%) as a pale yellow/white chalky powder. $\mathrm{mp}>300{ }^{\circ} \mathrm{C}$ (lit. $\left.{ }^{17},>300{ }^{\circ} \mathrm{C}\right) .{ }^{1} \mathrm{H} \mathrm{NMR}\left(250.1 \mathrm{MHz}, \mathrm{DMSO}-d_{6}\right): \delta 7.34(\mathrm{~s}, 1 \mathrm{H}$, $\mathrm{CH}), 7.89(\mathrm{~s}, 1 \mathrm{H}, \mathrm{CH})$, not observed $\left(\mathrm{CNO}_{2}\right)$. MS (Cl) $\mathrm{m} / \mathrm{z}(\%) 112\left(\mathrm{M}^{-}, 100\right)$.

4(5)-Nitroimidazole lithium salt (12). A solution of $\mathrm{LiOH} . \mathrm{H}_{2} \mathrm{O}(0.84 \mathrm{~g}, 20 \mathrm{mmol})$ in $\mathrm{MeOH}(10 \mathrm{~mL})$ was added dropwise to a stirred mixture of 4-nitroimidazole $(2.26 \mathrm{~g}, 20 \mathrm{mmol})$ and $\mathrm{MeOH}(20 \mathrm{~mL})$ at rt. After stirring $(30$ $\mathrm{min}$ ) the clear dark orange solution was evaporated under reduced pressure to give a solid that was dried under high vacuum to give compound 12 (2.33 g, 98\%) as an orange/brown solid. $\mathrm{mp}>300{ }^{\circ} \mathrm{C}$. ${ }^{1} \mathrm{H}$ NMR (DMSO$\left.d_{6}, 250.1 \mathrm{MHz}\right): \delta 7.34(\mathrm{~s}, 1 \mathrm{H}, \mathrm{CH}), 7.89(\mathrm{~s}, 1 \mathrm{H}, \mathrm{CH}) .{ }^{13} \mathrm{C} \mathrm{NMR}\left(62.9 \mathrm{MHz}, \mathrm{DMSO}-d_{6}\right): \delta 127.4(\mathrm{CH}), 142.7(\mathrm{CH})$, not observed $\left(\mathrm{CNO}_{2}\right)$. MS (Cl) $\mathrm{m} / \mathrm{z}(\%) 112\left(\mathrm{M}^{-}, 100\right)$.

4(5)-Nitroimidazole sodium salt (13). A solution of $\mathrm{NaOH}(1.6 \mathrm{~g}, 40 \mathrm{mmol})$ in $\mathrm{MeOH}(10 \mathrm{~mL})$ was added dropwise to a stirred mixture of 4-nitroimidazole $(4.5 \mathrm{~g}, 40 \mathrm{mmol})$ and $\mathrm{MeOH}(40 \mathrm{~mL})$ at $\mathrm{rt}$. After stirring (10 $\mathrm{min})$, a trace of insoluble material was filtered off, the clear yellow solution was evaporated under reduced pressure and the residue recrystallised from EtOH to give compound 13 (4.2 g, 79\%) as yellow crystals. mp $>300{ }^{\circ} \mathrm{C}$ (lit. $\left.{ }^{17},>300{ }^{\circ} \mathrm{C}\right) .{ }^{1} \mathrm{H}$ NMR $\left(250.1 \mathrm{MHz}, \mathrm{DMSO}-d_{6}\right): \delta 7.14(\mathrm{~s}, 1 \mathrm{H}, \mathrm{CH}), 7.75(\mathrm{~s}, 1 \mathrm{H}, \mathrm{CH}) .{ }^{13} \mathrm{C} \mathrm{NMR}$ (DMSO- $d_{6}$ $62.9 \mathrm{MHz}): \delta 131.0(\mathrm{CH}), 145.6(\mathrm{CH})$, not observed $\left(\mathrm{CNO}_{2}\right) . \mathrm{MS}(\mathrm{Cl}) \mathrm{m} / \mathrm{z}(\%) 112\left(\mathrm{M}^{-}, 100\right)$.

4(5)-Nitroimidazole cesium salt (14). Powdered 4-nitroimidazole (15.0 g, $133 \mathrm{mmol}$ ) was added to $\mathrm{MeOH}$ (150 $\mathrm{mL}$ ) and stirred at rt. An aqueous solution of $\mathrm{CsOH}(23.2 \mathrm{~mL} 50 \% \mathrm{w} / \mathrm{w}, 133 \mathrm{mmol})$ was added dropwise with stirring $(30 \mathrm{~min})$. After further stirring $(30 \mathrm{~min}$ ) the clear yellow solution was filtered, evaporated under reduced pressure and dried under high vacuum. Recrystallization of the resulting solid from isopropanol gave compound 14 (27.5 g, 73\%) as yellow/orange crystals. $\mathrm{mp}>300{ }^{\circ} \mathrm{C} .{ }^{1} \mathrm{H}$ NMR (DMSO- $d_{6}, 250.1 \mathrm{MHz}$ ): $\delta 7.09$ (s, $1 \mathrm{H}, \mathrm{CH}), 7.71(\mathrm{~s}, 1 \mathrm{H}, \mathrm{CH}) .{ }^{13} \mathrm{C}$ NMR $\left(62.9 \mathrm{MHz}, \mathrm{DMSO}-d_{6}\right): \delta 132.6(\mathrm{CH}), 146.8(\mathrm{CH})$, not observed $\left(\mathrm{CNO}_{2}\right) . \mathrm{MS}$ (Cl) $m / z(\%) 112\left(\mathrm{M}^{-}, 100\right)$.

1-(2'-Deoxy-3',5'-di-O-toluoyl- $\beta$-D-ribofuranosyl)-5-nitroimidazole (15). A solution of the sugar 10 (5.0 g, 12.9 $\mathrm{mmol}$ ) in dry THF (500 $\mathrm{mL}$ ) was added dropwise $(30 \mathrm{~min})$ to a stirred mixture of 4(5)-nitroimidazole cesium salt $14(3.8 \mathrm{~g}, 15.5 \mathrm{mmol})$ and dry THF $(500 \mathrm{~mL})$ at $60{ }^{\circ} \mathrm{C}$. After stirring at this temperature $(2 \mathrm{~h})$ the reaction mixture was allowed to cool to $\mathrm{rt}$ and filtered through celite. The filtrate and washings were evaporated under reduced pressure. The crude mixture of isomers was dissolved in $\mathrm{CHCl}_{3}(50 \mathrm{~mL})$ and loaded onto a flash chromatography column (70 mm ID column, $150 \mathrm{~mm}$ silica, packed dry and degassed with a 1:1 mixture of Et ${ }_{2} \mathrm{O}$ and $\mathrm{CHCl}_{3}$ ) and eluted with a 1:1 mixture of $\mathrm{Et}_{2} \mathrm{O}$ and $\mathrm{CHCl}_{3}$. Evaporation of the collected fractions and drying under high vacuum gave compound $15(4.1 \mathrm{~g}, 68 \%)$ as a colourless solid. $\mathrm{mp} 159-160{ }^{\circ} \mathrm{C} . R_{f} 0.49\left(\mathrm{Et}_{2} \mathrm{O}-\mathrm{CHCl}_{3}\right.$ 1:1). IR ( $\left.\mathrm{cm}^{-1}\right)$ 3030, 2983, 1723, 1528, 1466, 1111, 1092, 752. ${ }^{1} \mathrm{H} \mathrm{NMR}\left(250.1 \mathrm{MHz}, \mathrm{CDCl}_{3}\right): \delta 2.42\left(\mathrm{~s}, 3 \mathrm{H}, \mathrm{CH}_{3}\right)$, $2.46\left(\mathrm{~s}, 3 \mathrm{H}, \mathrm{CH}_{3}\right), 2.52\left(\mathrm{~m}, 1 \mathrm{H}, 2^{\prime}-\mathrm{CH} \beta\right), 3.12$ (ddd, J 14.3, 5.8, $\left.2.8 \mathrm{~Hz}, 1 \mathrm{H}, 2^{\prime}-\mathrm{CH} \alpha\right), 4.74\left(\mathrm{~m}, 3 \mathrm{H}, 5^{\prime}-\mathrm{CH}_{2}\right.$ and $4^{\prime}-$ $\mathrm{CH}), 5.64\left(\mathrm{~m}, 1 \mathrm{H}, 3^{\prime}-\mathrm{CH}\right), 6.72\left(\mathrm{t}, J 6.3 \mathrm{~Hz}, 1 \mathrm{H}, 1^{\prime}-\mathrm{CH}\right), 7.24(\mathrm{~d}, J 8.3 \mathrm{~Hz}, 2 \mathrm{H}, 3,5$-tol-CH), $7.30(\mathrm{~d}, J 8.3 \mathrm{~Hz}, 2 \mathrm{H}, 3,5-$ tol-CH), 7.84 (d, J $8.0 \mathrm{~Hz}, 2 \mathrm{H}, 2,6$-tol-CH), 7.97 (d, J $8.0 \mathrm{~Hz}, 2 \mathrm{H}, 2,6$-tol-CH), 8.05 (d, J $1.3 \mathrm{~Hz}, 1 \mathrm{H}, 4-\mathrm{CH}), 8.11$ (d, J $1.3 \mathrm{~Hz}, 1 \mathrm{H}, 2-\mathrm{CH}) .{ }^{13} \mathrm{C} N M R\left(62.9 \mathrm{MHz}, \mathrm{CDCl}_{3}\right): \delta 21.74\left(\mathrm{CH}_{3}\right), 21.81\left(\mathrm{CH}_{3}\right), 40.90\left(2^{\prime}-\mathrm{CH}_{2}\right), 63.66\left(5^{\prime}-\mathrm{CH}_{2}\right), 74.2$ $\left(4^{\prime}-\mathrm{CH}\right), 84.00\left(3^{\prime}-\mathrm{CH}\right), 88.04\left(1^{\prime}-\mathrm{CH}\right), 126.1(\mathrm{C}), 126.2(\mathrm{C}), 129.18(\mathrm{CH}), 129.37(\mathrm{CH}), 129.44(\mathrm{CH}) 129.62(\mathrm{CH})$ 
134.33 (4-CH), 138.00 (5-CH), 138.13 (2-CH), 144.5 (C), 144.7 (C), 166.0 (CO), 166.1 (CO). MS (EI) m/z (\%) 465 $\left(\mathrm{M}^{+}\right.$, 2), 320 (1), 216 (28), 136 (28), 119 (100), 91 (53), 81 (93), 65 (18), 53 (14), 39 (13), 28 (13). Anal. Calcd for $\mathrm{C}_{24} \mathrm{H}_{23} \mathrm{~N}_{3} \mathrm{O}_{7}: \mathrm{C}, 61.9 ; \mathrm{H}, 5.0 ; \mathrm{N}, 9.0$. Found: $\mathrm{C}, 61.7 ; \mathrm{H}, 4.9 ; \mathrm{N}, 8.8$. Evaporation of subsequent fractions gave compound $16(1.2 \mathrm{~g}, 20 \%)$ as a white solid. Analysis as for the following method for preparation of compounds 16 and 18.

1-(2'-Deoxy-3',5'-di-O-toluoyl- $\beta$-D-ribofuranosyl)-4-nitroimidazole (16) and 1-(2'-Deoxy-3',5'-di-O-toluoyl- $\alpha$ D-ribofuranosyl)-4-nitroimidazole (18). Method A: Sodium hydride 60\% in oil (0.61 g, $15.2 \mathrm{mmol})$ was added to a solution of 4-nitroimidazole $5(2.0 \mathrm{~g}, 17.7 \mathrm{mmol})$ in dry MeCN $(40 \mathrm{~mL})$ under argon. After stirring at rt (15 min), Hoffer's sugar 10 (6.0 g, $15.4 \mathrm{mmol})$ was added and stirring continued $(1 \mathrm{~h})$. The reaction mixture was then filtered and the combined filtrate and washings were evaporated under reduced pressure and dried under high vacuum to yield a crude product mixture. Flash chromatography (EtOAc-Hexane 1:1) was used to separate this mixture into the isomeric title compounds $16(0.82 \mathrm{~g}, 28 \%)$ and $18(0.47 \mathrm{~g}, 17 \%)$. Analysis as for method B. Method B: The silver salt 11 (4.7 g, $21.2 \mathrm{mmol})$ and Hoffer's sugar 10 (8.23 g, $21.2 \mathrm{mmol})$ in xylene (120 mL) were heated under reflux $\left(140^{\circ} \mathrm{C}\right)$ with stirring $(6 \mathrm{~h})$. After cooling and standing overnight, the reaction mixture was filtered under reduced pressure and the solid washed well with $\mathrm{CHCl}_{3}$. The filtrate and washings were removed at up to $60{ }^{\circ} \mathrm{C}$ under high vacuum to yield the crude reaction mixture. Flash column chromatography (EtOAc-hexanes, 1:1) was used to isolate two isomeric products, which were identified as compounds 16 and 18. The faster running compound was identified as compound $16(3.01 \mathrm{~g}, 31 \%)$ as fine colourless crystals. mp 116-117.5 ${ }^{\circ} \mathrm{C} . R_{f} 0.40$ (EtOAc-hexanes, 1:1). ${ }^{1} \mathrm{H} \mathrm{NMR}\left(250.1 \mathrm{MHz}, \mathrm{CDCl}_{3}\right): \delta 2.41\left(\mathrm{~s}, 3 \mathrm{H}, \mathrm{CH}_{3}\right), 2.44\left(\mathrm{~s}, 3 \mathrm{H}, \mathrm{CH}_{3}\right)$,

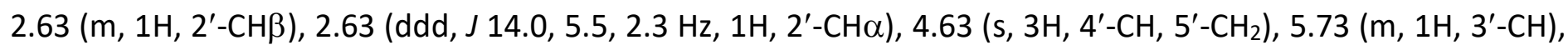
6.17 (dd, J 7.8, $\left.5.6 \mathrm{~Hz}, 1 \mathrm{H}, 1^{\prime}-\mathrm{CH}\right), 7.24(2 \mathrm{x} \mathrm{d}, J 8.0 \mathrm{~Hz}, 4 \mathrm{H}, 3,5-\mathrm{tol}-\mathrm{CH}), 7.64$ (d, J $\left.1.3 \mathrm{~Hz}, 1 \mathrm{H}, 2-\mathrm{CH}\right), 7.91(\mathrm{~d}, J 1.3$ $\mathrm{Hz}, 1 \mathrm{H}, 5-\mathrm{CH}) .7 .92\left(2 \mathrm{xd}, J 8.0 \mathrm{~Hz}, 4 \mathrm{H}, 2,6\right.$-tol-CH). ${ }^{13} \mathrm{C} \mathrm{NMR}\left(62.9 \mathrm{MHz}, \mathrm{CDCl}_{3}\right): \delta 21.74\left(\mathrm{CH}_{3}\right), 21.80\left(\mathrm{CH}_{3}\right), 39.86$ $\left(2^{\prime}-\mathrm{CH}_{2}\right), 63.58\left(5^{\prime}-\mathrm{CH}\right), 74.65\left(3^{\prime}-\mathrm{CH}\right), 83.82\left(4^{\prime}-\mathrm{CH}\right), 87.27\left(1^{\prime}-\mathrm{CH}\right), 116.79(5-\mathrm{CH}), 126.06(\mathrm{C}), 126.24(\mathrm{C}), 129.40$ $(\mathrm{CH}), 129.53(\mathrm{CH}), 129.67(\mathrm{CH}), 129.80(\mathrm{CH}), 134.28(2-\mathrm{CH}), 144.67(\mathrm{C}), 144.80(\mathrm{C}), 148.49\left(4-\mathrm{CNO}_{2}\right), 165.85$ (CO), 166.13 (CO). The slower running compound was isolated and identified as compound $18(1.34 \mathrm{~g}, 14 \%)$ as an off-white glassy solid. $R_{f} 0.27$ (EtOAc-hexanes, $\left.1: 1\right)$. ${ }^{1} \mathrm{H} \mathrm{NMR}\left(250.1 \mathrm{MHz}, \mathrm{CDCl}_{3}\right): \delta 2.38\left(\mathrm{~s}, 3 \mathrm{H}, \mathrm{CH}_{3}\right), 2.41(\mathrm{~s}$, $\left.3 \mathrm{H}, \mathrm{CH}_{3}\right), 2.84-2.99\left(2 \times \mathrm{m}, 2 \mathrm{H}, 2^{\prime}-\mathrm{CH}_{2}\right), 4.53\left(\mathrm{~s}, 2 \mathrm{H}, 5^{\prime}-\mathrm{CH}_{2}\right), 5.01$ (dd, J 5.2, $\left.4.2 \mathrm{~Hz}, 1 \mathrm{H}, 4^{\prime}-\mathrm{CH}\right), 5.64\left(\mathrm{~m}, 1 \mathrm{H}, 3^{\prime}-\right.$ $\mathrm{CH}), 6.46$ (d, 1H, J $\left.5.3 \mathrm{~Hz}, 1^{\prime}-\mathrm{CH}\right), 7.26$ (d, J $\left.8.2 \mathrm{~Hz}, 2 \mathrm{H}, 3,5-\mathrm{tol}-\mathrm{CH}\right), 7.37$ (d, J $8.2 \mathrm{~Hz}, 2 \mathrm{H}, 3,5$-tol-CH), 7.66 (d, J $8.2 \mathrm{~Hz}, 2 \mathrm{H}, 2,6$-tol-CH), 7.95 (d, J $8.2 \mathrm{~Hz}, 2 \mathrm{H}, 2,6$-tol-CH), $8.13(\mathrm{~s}, 1 \mathrm{H}, 2-\mathrm{CH}), 8.62(\mathrm{~s}, 1 \mathrm{H}, 5-\mathrm{CH}) .{ }^{13} \mathrm{C} \mathrm{NMR}\left(\mathrm{CDCl}_{3}\right.$, $62.9 \mathrm{MHz}): \delta 21.37\left(2 \times \mathrm{CH}_{3}\right), 38.85\left(2^{\prime}-\mathrm{CH}_{2}\right), 64.20\left(5^{\prime}-\mathrm{CH}\right), 74.99\left(3^{\prime}-\mathrm{CH}\right), 84.36\left(4^{\prime}-\mathrm{CH}\right), 88.35\left(1^{\prime}-\mathrm{CH}\right), 119.53$ (5-CH), $126.51(\mathrm{C}), 126.76(\mathrm{C}), 129.38(\mathrm{CH}), 129.42(\mathrm{CH}), 129.59(\mathrm{CH}), 129.61(\mathrm{CH}) 135.72(2-\mathrm{CH}), 144.20(\mathrm{C})$, 144.33 (C), $147.47\left(4-\mathrm{CNO}_{2}\right), 165.20$ (CO), 165.70 (CO).

1-(2'-Deoxy- $\beta$-D-ribofuranosyl)-4-nitroimidazole (19). Aqueous methylamine $(40 \%, 8.0 \mathrm{~mL})$ was added to a solution of compound $16(0.78 \mathrm{~g}, 1.7 \mathrm{mmol})$ in $\mathrm{MeOH}(30 \mathrm{~mL})$, and the resulting solution was left to stand at $\mathrm{rt}$ overnight. The solution was then evaporated under reduced pressure and the residue purified by flash chromatography (EtOAc-MeOH, 9:1) to yield compound 19 (0.34 g, 87\%). An analytical sample was obtained by recrystallisation from EtOAc-MeOH (4:1) as a colourless solid. $R_{f} 0.42$ (EtOAc-MeOH, 9:1). ${ }^{1} \mathrm{H} \mathrm{NMR}(400.1 \mathrm{MHz}$, DMSO- $\left.d_{6}\right): \delta 2.30\left(\mathrm{ddd}, 1 \mathrm{H}, 2^{\prime}-\mathrm{CH} \alpha\right), 2.30\left(\mathrm{~m}, 1 \mathrm{H}, 2^{\prime}-\mathrm{CH} \beta\right), 3.56\left(\mathrm{~m}, 2 \mathrm{H}, 5^{\prime}-\mathrm{CH}_{2}\right), 3.88\left(\mathrm{~d}, 1 \mathrm{H}, 44^{\prime}-\mathrm{CH}\right), 4.34(\mathrm{~s}, 1 \mathrm{H}$, $\left.3^{\prime}-\mathrm{CH}\right), 5.08\left(\mathrm{t}, 1 \mathrm{H}, 5^{\prime}-\mathrm{OH}\right), 5.37\left(\mathrm{~d}, 1 \mathrm{H}, 3^{\prime}-\mathrm{OH}\right), 6.14\left(\mathrm{dd}, 1 \mathrm{H}, 1^{\prime}-\mathrm{CH}\right), 8.11(\mathrm{~d}, 1 \mathrm{H}, 2-\mathrm{CH}), 8.59(\mathrm{~d}, 1 \mathrm{H}, 5-\mathrm{CH}) .{ }^{13} \mathrm{C}$ NMR (62.9 MHz, DMSO- $\left.d_{6}\right): \delta 41.45\left(2^{\prime}-\mathrm{CH}_{2}\right), 61.49\left(5^{\prime}-\mathrm{CH}_{2}\right), 70.61\left(3^{\prime}-\mathrm{CH}\right), 87.16\left(4^{\prime}-\mathrm{CH}\right), 88.56\left(1^{\prime}-\mathrm{CH}\right), 119.58$ $(5-\mathrm{CH}), 136.26(2-\mathrm{CH}), 147.40\left(4-\mathrm{CNO}_{2}\right)$.

1-(2'-Deoxy- $\alpha$-D-ribofuranosyl)-4-nitroimidazole (20). Using aqueous methylamine, compound 20 (1.03 g, 84\%), was prepared from compound 18 using the above procedure for the preparation of compound 19 from compound 16. $\mathrm{mp} 118.5-119.5^{\circ} \mathrm{C} . R_{f} 0.27$ (EtOAc-MeOH, 9:1). ${ }^{1} \mathrm{H} \mathrm{NMR}\left(400.1 \mathrm{MHz}, \mathrm{DMSO}-d_{6}\right): \delta 1.94$ (d, $1 \mathrm{H}$, 
2'-CH), 2.69 (q, 1H, 2'-CH), $3.43\left(\mathrm{~m}, 2 \mathrm{H}, 5^{\prime}-\mathrm{CH}_{2}\right) 4.16\left(\mathrm{~s}, 1 \mathrm{H}, 4^{\prime}-\mathrm{CH}\right), 4.31\left(\mathrm{~s}, 1 \mathrm{H}, 3^{\prime}-\mathrm{CH}\right), 4.91\left(\mathrm{t}, 1 \mathrm{H}, 5^{\prime}-\mathrm{OH}\right), 5.52$ (s, 1H, 3'-OH), 6.15 (d, 1H, J $\left.7.1 \mathrm{~Hz}, 1^{\prime}-\mathrm{CH}\right), 8.06(\mathrm{~s}, 1 \mathrm{H}, 2-\mathrm{CH}), 8.56(\mathrm{~s}, 1 \mathrm{H}, 5-\mathrm{CH}) .{ }^{13} \mathrm{C}$ NMR (62.9 MHz, DMSO$\left.d_{6}\right): \delta 41.08\left(2^{\prime}-\mathrm{CH}_{2}\right), 61.76\left(5^{\prime}-\mathrm{CH}_{2}\right), 70.83\left(3^{\prime}-\mathrm{CH}\right), 87.95\left(4^{\prime}-\mathrm{CH}\right), 89.83\left(1^{\prime}-\mathrm{CH}\right), 120.01(5-\mathrm{CH}), 136.48(2 \mathrm{CH})$, $147.36(4-C)$.

5-Amino-1-(2'-deoxy-3',5'-di-O-p-toluoyl- $\beta$-D-ribofuranosyl)-imidazole (21). 1-(2'-Deoxy-3',5'-di-O-toluoyl- $\beta$ D-ribofuranosyl)-4-nitroimidazole $15(50 \mathrm{mg}, 0.11 \mathrm{mmol}), 5 \% \mathrm{Pd}$ on C (50 mg) and THF- $d_{8}(1.0 \mathrm{~mL})$ were vigorously shaken with $\mathrm{H}_{2}$ at atmospheric pressure and rt. After $2.5 \mathrm{~h}$ TLC analysis indicated that no starting material remained. The reaction mixture was filtered through a THF- $d_{8}$ wetted bed of pre-dried sand and celite, in a Pasteur pipette, using a slight argon pressure into a dry, argon-purged NMR tube. The filter bed was washed with THF- $d_{8}(2 \times 0.25 \mathrm{~mL})$. The clear pale yellow solution of compound 21 was analysed immediately by ${ }^{1} \mathrm{H}$ NMR. $R_{f} 0.57$ (EtOAc-MeOH-conc. aq NH $\left.3,10: 1: 1\right) .{ }^{1} \mathrm{H}$ NMR (THF- $\left.d_{8}\right): \delta 2.37(\mathrm{~s}, 3 \mathrm{H}),, 2.39\left(\mathrm{~s}, 3 \mathrm{H}, \mathrm{CH}_{3}\right), 2.55$ (ddd, $\left.1 \mathrm{H}, 2^{\prime}-\mathrm{CH \alpha}\right), 2.97\left(\mathrm{~m}, 1 \mathrm{H}, 2^{\prime}-\mathrm{CH} \beta\right), 4.01$ (br s, 2H, NH 2$), 4.52\left(2 \mathrm{x} \mathrm{m}, 3 \mathrm{H}, 5^{\prime}-\mathrm{CH}_{2}\right.$ and 4'-CH), $5.63\left(\mathrm{~m}, 1 \mathrm{H}, 33^{\prime}-\right.$ $\mathrm{CH}), 6.07\left(\mathrm{dd}, 1 \mathrm{H}, J\right.$ 8.9, $\left.5.4 \mathrm{~Hz}, 1^{\prime}-\mathrm{CH}\right), 6.15(\mathrm{~s}, 1 \mathrm{H}, 4 \mathrm{H}), 7.26(\mathrm{~m}, 5 \mathrm{H}, 3,5$-tol-CH and 2H), 7.92 (d, 4H, 2,6-tol$\mathrm{CH})$.

5-Amino-4-(2,2-dicyanovinyl)-1-(2'-deoxy-3',5'-di-O-p-toluoyl- $\beta$-D-ribofuranosyl)-imidazole (22). 1-(2'-Deoxy3',5'-di-O-toluoyl- $\beta$-D-ribofuranosyl)-4-nitroimidazole 15 (3.27 g, $7.03 \mathrm{mmol}$ ), 5\% Pd on C (3.27 g) and dry THF $(150 \mathrm{~mL})$ were vigorously shaken with $\mathrm{H}_{2}$ at atmospheric pressure and $\mathrm{rt}$. After $2 \mathrm{~h}$, a sample was removed and TLC analysis indicated a spot due to compound $\mathbf{2 1}$ and no starting material. The reaction flask was evacuated and purged with argon. The product mixture was then filtered through a bed of dry celite (acid washed) in an enclosed system using argon pressure (2 to 4 psi) into a dry, argon-purged receiver flask containing ethoxymethylene malononitrile $(1.46 \mathrm{~g}, 13.52 \mathrm{mmol})$. The filter bed was washed with dry THF $(3 \times 20 \mathrm{~mL})$, again using argon pressure, and the washings were combined with the filtered reaction mixture in the receiver flask. The reaction was stirred at $50{ }^{\circ} \mathrm{C}$ under argon, overnight to give a dark brown solution. The THF was removed under vacuum at room temperature and chilled ethyl acetate $(20 \mathrm{~mL})$ was added to the residue. The product was filtered off and washed with chilled ethyl acetate $(2 \times 20 \mathrm{~mL})$ to yield compound 22 (1.95 g, 54\%) as a yellow solid. $\mathrm{mp} 194-196^{\circ} \mathrm{C}$. $R_{f} 0.73$ (EtOAc-MeOH-conc. aq NH$, 10: 1: 1$ ). IR (cm ${ }^{-1}$ ) 3348, 2924, 2218, 1715, 1586, 1540, 1354, 1283, 1266, 1112, $752 \mathrm{~cm}^{-1} .{ }^{1} \mathrm{H}$ NMR $\left(250.1 \mathrm{MHz}, \mathrm{DMSO}-d_{6}\right): \delta 2.39\left(\mathrm{~s}, 3 \mathrm{H}, \mathrm{CH}_{3}\right), 2.41(\mathrm{~s}, 3 \mathrm{H}$, $\left.\mathrm{CH}_{3}\right), 2.72\left(\mathrm{~m}, 1 \mathrm{H}, 2^{\prime}-\mathrm{CH} \alpha\right), 2.86\left(\mathrm{~m}, 1 \mathrm{H}, 2^{\prime}-\mathrm{CH} \beta\right), 4.53\left(\mathrm{~m}, 3 \mathrm{H}, 5^{\prime}-\mathrm{CH}_{2}\right.$ and $\left.4^{\prime}-\mathrm{CH}\right), 5.63\left(\mathrm{~m}, 1 \mathrm{H}, 3^{\prime}-\mathrm{CH}\right), 6.09(\mathrm{dd}$, $1 \mathrm{H}, J$ 8.5, $\left.5.7 \mathrm{~Hz}, 1^{\prime}-\mathrm{CH}\right), 7.34$ (d, J $8.3 \mathrm{~Hz}, 2 \mathrm{H}, 3,5$-tol-CH), 7.38 (d, J $8.3 \mathrm{~Hz}, 2 \mathrm{H}, 3,5$-tol-CH), 7.72 (br s, 2H, NH ), $7.79(\mathrm{~s}, 1 \mathrm{H}, \mathrm{C}=\mathrm{CH}), 7.83(\mathrm{~s}, 1 \mathrm{H}, 2-\mathrm{CH}), 7.86$ (d, J $8.3 \mathrm{~Hz}, 2 \mathrm{H}, 2,6-$ tol-CH), 7.95 (d, J $8.3 \mathrm{~Hz}, 2 \mathrm{H}, 2,6-$ tol-CH). ${ }^{13} \mathrm{C}$ NMR (62.9 MHz, DMSO-d $\left.\left.)_{6}\right): \delta 21.17\left(\mathrm{CH}_{3}\right), 21.21\left(\mathrm{CH}_{3}\right), 35.88\left(2^{\prime}-\mathrm{CH}_{2}\right), 58.63(\mathrm{C}(\mathrm{CN}))_{2}\right), 63.96\left(5^{\prime}-\mathrm{CH}_{2}\right), 74.72\left(4^{\prime}-\right.$ $\mathrm{CH}), 81.69\left(3^{\prime}-\mathrm{CH}\right), 82.81\left(1^{\prime}-\mathrm{CH}\right), 116.20(4-\mathrm{C}), 117.99(\mathrm{CN}), 118.56(\mathrm{CN}), 126.43(2 \times \mathrm{C}), 129.29(\mathrm{CH}), 129.38$ $(\mathrm{CH}), 129.50(\mathrm{CH}), 134.21(2-\mathrm{CH}), 143.53(\mathrm{C}=\mathrm{CH}), 143.84(\mathrm{C}), 144.08(\mathrm{C}), 150.02(5-\mathrm{C}), 165.12(\mathrm{CO}), 165.40(\mathrm{CO})$. MS (ES): $m / z(\%) 511\left(\mathrm{M}^{+}, 7\right), 353$ (7), 159 (41), 119 (68), 91 (23), 81 (100).

5-Amino-6-cyano-3-(2'-deoxy-3',5'-di-O-p-toluoyl- $\beta$-D-ribofuranosyl)-imidazo[4,5-b]pyridine (23). Method A. Dowex 1X2-200 ion exchange resin (303 mg) was stirred at $65{ }^{\circ} \mathrm{C}(3 \mathrm{~h})$ with amine $22(2.0 \mathrm{~g}, 3.91 \mathrm{mmol})$ in acetonitrile $(54 \mathrm{~mL})$. The reaction mixture was filtered and the filtrate concentrated under vacuum and dried to give the compound $23(1.65 \mathrm{~g}, 83 \%)$ as a yellow solid. mp 115-116 ${ }^{\circ} \mathrm{C} . R_{f} 0.55$ (Et $\left.{ }_{2} \mathrm{O}-\mathrm{MeOH} 9: 1\right) . \mathrm{IR}\left(\mathrm{cm}^{-1}\right)$ $3347,3193,3044,2947,2914,2210,1712,1612,1260,1166,1087,750 .{ }^{1} \mathrm{H} \mathrm{NMR}\left(400.1 \mathrm{MHz}, \mathrm{CDCl}_{3}\right): \delta 2.38(\mathrm{~s}$, $\left.3 \mathrm{H}, \mathrm{CH}_{3}\right), 2.43\left(\mathrm{~s}, 3 \mathrm{H}, \mathrm{CH}_{3}\right), 2.73\left(\mathrm{ddd}, J 14.1,6.7,2.5 \mathrm{~Hz}, 1 \mathrm{H}, 2^{\prime}-\mathrm{CH} \alpha\right), 2.89\left(\mathrm{~m}, 1 \mathrm{H}, 2^{\prime}-\mathrm{CH} \beta\right), 4.66\left(\mathrm{~m}, 3 \mathrm{H}, 5^{\prime}-\mathrm{CH}_{2}\right.$ and 4'-CH), 5.80 (br s, 2H, NH$\left.)_{2}\right), 5.35\left(\mathrm{~m}, 1 \mathrm{H}, 3^{\prime}-\mathrm{CH}\right), 6.29\left(\mathrm{dd}, J 8.9,5.5 \mathrm{~Hz}, 1 \mathrm{H}, 1^{\prime}-\mathrm{CH}\right), 7.20(\mathrm{~d}, J 8.0 \mathrm{~Hz}, 2 \mathrm{H}, 3,5-$ tol-CH), 7.28 (d, J $8.0 \mathrm{~Hz}, 2 \mathrm{H}, 3,5$-tol-CH), 7.80 (d, J $8.0 \mathrm{~Hz}, 2 \mathrm{H}, 2,6$-tol-CH), 7.95 (d, J $8.0 \mathrm{~Hz}, 2 \mathrm{H}, 2,6$-tol-CH), $7.98(\mathrm{~s}, 1 \mathrm{H}, 7-\mathrm{CH}), 8.32(\mathrm{~s}, 1 \mathrm{H}, 2-\mathrm{CH}) .{ }^{13} \mathrm{C} \mathrm{NMR}\left(101.6 \mathrm{MHz}, \mathrm{CDCl}_{3}\right): \delta 21.72\left(\mathrm{CH}_{3}\right), 21.77\left(\mathrm{CH}_{3}\right), 36.90\left(2^{\prime}-\mathrm{CH}_{2}\right)$, $63.83\left(5^{\prime}-\mathrm{CH}_{2}\right), 75.07$ (4'-CH), $82.66\left(3^{\prime}-\mathrm{CH}\right), 84.87\left(1^{\prime}-\mathrm{CH}\right), 87.89(6-\mathrm{C}), 117.73\left(\mathrm{CNH}_{2}\right), 126.43(\mathrm{C}), 126.61(\mathrm{C})$, 
128.53 (C) 129.30 (2 x CH), 129.65 (CH), $129.79(\mathrm{CH}), 133.89$ (2-CH), 144.25 (C), 144.54 (C), 148.05 (C), 156.74 (5-C), 165.93 (CO), 166.36 (CO).

Method B. Sodium hydride $60 \%$ in oil $(250 \mathrm{mg}, 6.3 \mathrm{mmol})$ was added to a solution of 5-aminoimidazo[4,5,b]pyrimidine-6-carbonitrile $25^{31}(1.0 \mathrm{~g}, 6.3 \mathrm{mmol})$ in dry THF $(20 \mathrm{~mL})$ under argon with stirring $\left(55^{\circ} \mathrm{C}\right)$. Hoffer's sugar 10 (2.44 g, $6.3 \mathrm{mmol})$ in THF $(25 \mathrm{~mL})$ was then added dropwise (15 $\mathrm{min})$ and stirring continued ( $2 \mathrm{~h})$. The reaction mixture was filtered, and insoluble salts were washed with THF. The combined filtrate and washings were evaporated under reduced pressure and dried under high vacuum to give the crude product mixture (2.77 g) of compound 23 and isomer 26 (1:2). Flash chromatography (EtOAc-petroleum ether bp 60-80 ${ }^{\circ} \mathrm{C}, 2: 1$ ) was used to remove faster running impurities then further elution (EtOAc) was used to isolate compound $\mathbf{2 3}$ $(0.61 \mathrm{~g}, 18 \%)$ as fine yellow needles; analysis as for method A. Further elution (EtOAc-MeOH, 9:1) was used to isolate isomer $26(0.71 \mathrm{mg}, 22 \%)$ as a green, glassy solid; mp 107-108 ${ }^{\circ} \mathrm{C} ; R_{f} 0.22$ (EtOAc-MeOH 9:1). IR (cm-1) $3465,3356,3199,3032,2947,2913,2213,1712,1606,1263,1184,1093,753 ;{ }^{1} \mathrm{H} \mathrm{NMR}\left(400.1 \mathrm{MHz} \mathrm{CDCl}_{3}\right): \delta$ $2.39\left(\mathrm{~s}, 3 \mathrm{H}, \mathrm{CH}_{3}\right), 2.43\left(\mathrm{~s}, 3 \mathrm{H}, \mathrm{CH}_{3}\right), 2.73\left(\mathrm{ddd}, J 10.6,6.1,2.5 \mathrm{~Hz}, 1 \mathrm{H}, 2^{\prime}-\mathrm{CH} \alpha\right), 3.18\left(\mathrm{~m}, 1 \mathrm{H}, 2^{\prime}-\mathrm{CH} \beta\right), 4.65(\mathrm{~m}, 2 \mathrm{H}$, $\left.5^{\prime}-\mathrm{CH}_{2}\right), 4.86\left(\mathrm{~m}, 1 \mathrm{H}, 4^{\prime}-\mathrm{CH}\right), 5.25\left(\mathrm{br} \mathrm{s}, 2 \mathrm{H}, \mathrm{NH}_{2}\right), 5.35\left(\mathrm{~m}, 1 \mathrm{H}, 3^{\prime}-\mathrm{CH}\right), 6.43\left(\mathrm{dd}, J 8.0,7.1 \mathrm{~Hz}, 1 \mathrm{H}, 1^{\prime}-\mathrm{CH}\right), 7.21(\mathrm{~d}$, J $8.1 \mathrm{~Hz}, 2 \mathrm{H}, 3,5$-tol-CH), $7.27(\mathrm{~d}, J 8.1 \mathrm{~Hz}, 2 \mathrm{H}, 3,5$-tol-CH), 7.88 (d, J $8.1 \mathrm{~Hz}, 2 \mathrm{H}, 2,6$-tol-CH), $7.97(\mathrm{~d}, J 8.1 \mathrm{~Hz}, 2 \mathrm{H}$, 2,6-tol-CH), $8.03(\mathrm{~s}, 1 \mathrm{H}, 2-\mathrm{CH}), 8.04(\mathrm{~s}, 1 \mathrm{H}, 7-\mathrm{CH}) .{ }^{13} \mathrm{C} \mathrm{NMR}\left(101.6 \mathrm{MHz}, \mathrm{CDCl}_{3}\right): \delta 21.71\left(\mathrm{CH}_{3}\right), 21.77\left(\mathrm{CH}_{3}\right)$, $38.90\left(2^{\prime}-\mathrm{CH}_{2}\right), 63.68\left(5^{\prime}-\mathrm{CH}_{2}\right), 74.45\left(4^{\prime}-\mathrm{CH}\right), 83.03$ (3'-CH), $86.23\left(1^{\prime}-\mathrm{CH}\right), 88.09$ (6-C), 118.08 (CN), $126.15(\mathrm{C})$, $126.24(\mathrm{C}), 128.53$ (C) $129.03(2-\mathrm{CH}) 129.35(\mathrm{CH}), 129.43(\mathrm{CH}), 129.46(\mathrm{CH}), 129.79(\mathrm{CH}), 130.04(7-\mathrm{CH}), 144.46$ (C), 144.73 (C), 157.28 (C), 157.70 (C), 165.93 (CO), 166.07 (CO), 175.45 (5-C).

Method C. Sodium hydride $60 \%$ in oil $(0.25 \mathrm{mg}, 6.3 \mathrm{mmol})$ was added to a solution of 5-aminoimidazo[4,5,b]pyridine-6-carbonitrile $25^{31}(1.0 \mathrm{~g}, 6.3 \mathrm{mmol})$ in dry THF $(20 \mathrm{~mL})$ under argon. After stirring the mixture at rt (15 min), Hoffer's sugar 10 (2.44 g, $6.3 \mathrm{mmol}$ ) was added and stirring continued $(2 \mathrm{~h})$. The reaction mixture was filtered, insoluble salts were washed with THF. The combined filtrate and washings were evaporated under reduced pressure and dried under high vacuum to give the crude product mixture $(2.77 \mathrm{~g})$ of compound 23 and isomer 26 in near 1:1 ratio, as judged by proton NMR analysis. Flash chromatography (EtOAc-petroleum ether bp $60-80^{\circ} \mathrm{C}, 2: 1$ ) was used to remove faster running impurities then further elution (EtOAc) was used to isolate compound $23(0.46 \mathrm{~g}, 14 \%)$ as fine yellow needles; analysis as for method $\mathrm{A}$.

5-Amino-6-cyano-3-(2'-deoxy- $\beta$-D-ribofuranosyl)-imidazo[4,5-b]pyridine (24). Method A: Sodium hydroxide $(1.0 \mathrm{~g}, 25 \mathrm{mmol})$ in water $(5.0 \mathrm{~mL})$ was added to a stirred solution of amine $22(0.4 \mathrm{~g}, 0.78 \mathrm{mmol})$ in $\mathrm{MeOH}(40$ $\mathrm{mL}$ ). The reaction mixture was heated to reflux temperature. After $10 \mathrm{~min}$, TLC analysis indicated no starting material and one main spot was observed that was strongly fluorescent. The reaction mixture was cooled and neutralised to $\mathrm{pH} 7$ using citric acid $(20 \% \mathrm{w} / \mathrm{v}$ aq). The mixture was then filtered through a bed of acid-washed filter aid and the filter cake washed well with $\mathrm{MeOH}$. The filtrate and washings were evaporated under reduced pressure. The residue was re-dissolved in warm $\mathrm{MeOH}$, pre-adsorbed on silica and purified by flash chromatography (EtOAc-MeOH-conc. aq $\mathrm{NH}_{3}$ 15:1:1) to yield compound $24(0.19 \mathrm{~g}, 88 \%$ ) as a light yellow green powder. mp 162-163.5 ${ }^{\circ} \mathrm{C}$. $R_{f} 0.55$ (EtOAc-MeOH-conc. aq NH$, 5: 1: 1$ ). IR (cm ${ }^{-1}$ ) 3337, 3223, 2923, 2216, 1630, 1576, 1432, 1099, 940. ${ }^{1} \mathrm{H}$ NMR $\left(250.1 \mathrm{MHz}, \mathrm{DMSO}-d_{6}\right): \delta 2.23$ (ddd, J 9.0, 7.3, $\left.3.3 \mathrm{~Hz}, 1 \mathrm{H}, 2^{\prime}-\mathrm{CH} \alpha\right), 2.56$ $\left(\mathrm{m}, 1 \mathrm{H}, 2^{\prime}-\mathrm{CH} \beta\right), 3.52\left(\mathrm{~m}, 2 \mathrm{H}, 5^{\prime}-\mathrm{CH}_{2}\right), 3.82\left(\mathrm{~m}, 1 \mathrm{H}, 4^{\prime}-\mathrm{CH}\right), 4.36\left(\mathrm{~m}, 1 \mathrm{H}, 3^{\prime}-\mathrm{CH}\right), 4.95\left(\mathrm{t}, 1 \mathrm{H}, \mathrm{J} 5.3 \mathrm{~Hz}, 5^{\prime}-\mathrm{OH}\right), 5.31$ (d, $\left.1 \mathrm{H}, J 3.5 \mathrm{~Hz}, 3^{\prime}-\mathrm{OH}\right), 6.28$ (dd, 1H, J 7.8, $\left.6.6 \mathrm{~Hz}, 1^{\prime}-\mathrm{CH}\right), 6.80$ (br s, 2H, NH $\mathrm{NH}_{2}, 8.25$ (s, 1H, 7-CH), 8.42 (s, 1H, 2$\mathrm{CH}) .{ }^{13} \mathrm{C}$ NMR $\left(62.9 \mathrm{MHz}, \mathrm{DMSO}-d_{6}\right): \delta 39.51\left(2^{\prime}-\mathrm{CH}_{2}\right), 61.89\left(5^{\prime}-\mathrm{CH}_{2}\right), 70.99\left(4^{\prime}-\mathrm{CH}\right), 82.71\left(3^{\prime}-\mathrm{CH}\right), 86.26(6-\mathrm{C})$, $87.89\left(1^{\prime}-\mathrm{CH}\right), 117.73(\mathrm{CN}), 126.80$ (C), $134.37(2-\mathrm{CH}), 141.99(7-\mathrm{CH}), 148.67$ (C), 157.55 (5-C). MS (EI) m/z (\%) $275\left(\mathrm{M}^{+}\right.$, 84), 186 (100), 160 (100), 159 (100), 132 (35), $117(63), 99$ (32), 73 (50), 45 (39), 43 (36), $28(26)$. HRMS (Cl) $m / z\left[\mathrm{M}^{+}+\mathrm{Na}\right]$ calcd for $\mathrm{C}_{12} \mathrm{H}_{13} \mathrm{~N}_{5} \mathrm{O}_{3} \mathrm{Na}$ : 298.0916. Found: 298.0919 . 
Method B: Dowex 1X2-200 ion exchange resin (5.2 g) was stirred with aq $\mathrm{NaOH}(2 \mathrm{M}, 50 \mathrm{~mL})$ for $30 \mathrm{~min}$. The ion exchange resin was filtered off and washed with de-ionised water until the washings were neutral and then washed with $\mathrm{MeOH}(2 \times 40 \mathrm{~mL})$. Suction was applied for $30 \mathrm{~min}$ to dry the resin. The prepared resin was added to amine $22(2.8 \mathrm{~g}, 5.5 \mathrm{mmol})$ and $\mathrm{MeOH}(50 \mathrm{~mL})$, and the resultant mixture stirred $(3 \mathrm{~h})$ at rt. TLC analysis indicated that no starting material was present and only one fluorescent product spot was observed. The reaction mixture was filtered and the resin washed with hot $\mathrm{MeOH}\left(5 \times 50 \mathrm{~mL}, 50\right.$ to $\left.60{ }^{\circ} \mathrm{C}\right)$. The filtrate and washings were evaporated under reduced pressure and $\mathrm{Et}_{2} \mathrm{O}(50 \mathrm{~mL})$ was added to the residue. The insoluble material was filtered off, washed with $\mathrm{Et}_{2} \mathrm{O}(2 \times 50 \mathrm{~mL})$ and dried under high vacuum to give compound 22 $(1.33 \mathrm{~g}, 89 \%)$ as a light fawn-coloured solid. Analysis as for method $\mathrm{A}$.

5-Amino-3-(2'-deoxy- $\beta$-D-ribofuranosyl)-imidazo[4,5-b]pyridine-6-carboxamide (27). Method $\mathrm{A}: \mathrm{H}_{2} \mathrm{O}_{2}(2.3$ $\mathrm{mL}, 35 \% \mathrm{w} / \mathrm{w}, 23.0 \mathrm{mmol}$ ) was added to a stirred mixture of nitrile $24(0.63 \mathrm{~g}, 2.3 \mathrm{mmol})$ in conc. aq $\mathrm{NH}_{3}(25$ $\mathrm{mL}$ ). After stirring at $\mathrm{rt}(30 \mathrm{~min}$ ) the solution became clear. TLC analysis after $2 \mathrm{~h}$ indicated no starting material and only one product spot. The reaction mixture was partially evaporated under reduced pressure, and after removal of excess $\mathrm{NH}_{3}$ a precipitate formed. The volume was reduced to ca $10 \mathrm{~mL}$ and cooled in the fridge (2 h). The precipitate was filtered off, washed with $\mathrm{MeOH}(2 \times 5 \mathrm{~mL})$ and dried under high vacuum to give compound $27(0.53 \mathrm{~g}, 79 \%)$ as a very light yellow solid. $\mathrm{mp} 222-223.5^{\circ} \mathrm{C} . R_{f} 0.32$ (EtOAc-MeOH-conc. aq $\mathrm{NH}_{3}$,

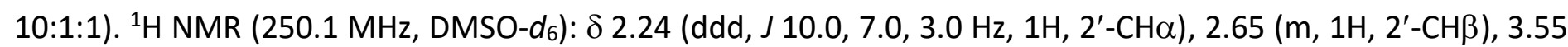
$\left(\mathrm{m}, 2 \mathrm{H}, 5^{\prime}-\mathrm{CH}_{2}\right), 3.84\left(\mathrm{~m}, 1 \mathrm{H}, 4^{\prime}-\mathrm{CH}\right), 4.39\left(\mathrm{~m}, 1 \mathrm{H}, 3^{\prime}-\mathrm{CH}\right), 5.00$ (br s, 1H, 5'-OH), 5.27 (br s, 1H, 3'-OH), 6.31 (dd, $1 \mathrm{H}$, J 7.8, $\left.6.0 \mathrm{~Hz}, 1^{\prime}-\mathrm{CH}\right), 7.31$ (s, 2H, NH ), 7.90 (br s, 1H, NH), 8.28 (s, 1H, 7-CH), 8.35 (1H, s, 2-CH), 10.20 (br s, $1 \mathrm{H}, \mathrm{NH}) .{ }^{13} \mathrm{C}$ NMR $\left(62.9 \mathrm{MHz}, \mathrm{DMSO}-d_{6}\right): \delta 39.18\left(2^{\prime}-\mathrm{CH}_{2}\right), 61.89\left(5^{\prime}-\mathrm{CH}_{2}\right), 70.88\left(4^{\prime}-\mathrm{CH}\right), 82.62\left(3^{\prime}-\mathrm{CH}\right), 87.55$ (1'-CH), 105.92 (C), 126.81 (C), 129.48 (2-CH), 140.8 (C), 147.47 (7-CH), 156.94 (C), 170.50 (CO). HRMS (CI): m/z $\left[\mathrm{M}^{+}+\mathrm{Na}\right]$ calcd for $\mathrm{C}_{12} \mathrm{H}_{15} \mathrm{~N}_{5} \mathrm{O}_{4} \mathrm{Na} ; 316.1022$. Found: 316.1017 .

Method B: Sodium hydroxide $(3.0 \mathrm{~g}, 75 \mathrm{mmol})$ in water $(15 \mathrm{~mL})$ was added to $\mathrm{MeOH}(80 \mathrm{~mL})$ followed by amine $22\left(1.2 \mathrm{~g}, 2.3 \mathrm{mmol}\right.$ ) and the mixture refluxed (3 h). TLC analysis (EtOAc-MeOH-conc. $\mathrm{NH}_{3}(\mathrm{aq})$ 5:1:1) showed compound $\mathbf{2 3}$ as a minor component with compound $\mathbf{2 7}$ as the major component accompanied by two minor spots of lower $R_{f}$ value. The reaction mixture was cooled, neutralized with citric acid ( $20 \% \mathrm{w} / \mathrm{v}$ aq) and filtered. The filtrate and $\mathrm{MeOH}$ washings were combined and evaporated under reduced pressure. The residue was preadsorbed on silica and separated by flash column chromatography (EtOAc-MeOH-conc. aq $\mathrm{NH}_{3}, 15: 1: 1$ ). Residual methyl toluate was removed by sublimation $\left(80^{\circ} \mathrm{C}, 3 \mathrm{~h}\right)$ under high vacuum. An initial fraction of nitrile 24 ( $0.20 \mathrm{~g}, 0.8 \mathrm{mmol})$ was recovered. The main fraction yielded compound $27(0.32 \mathrm{~g}, 67 \%$ based on recovered 24). Analysis as for method $A$.

3-(2'-Deoxy- $\beta$-D-ribofuranosyl)-8H-imidazo[4',5':5,6]-pyrido[2,3-d]pyrimidin-8-one (5). Sodium metal (0.35 g, $15.3 \mathrm{mmol}$ ) was dissolved in EtOH (pre-dried over $3 \AA$ molecular sieve) $(15 \mathrm{~mL})$ under argon. Carboxamide 27 $(0.45 \mathrm{~g}, 1.53 \mathrm{mmol})$ and ethyl formate $(1.1 \mathrm{~g}, 15.3 \mathrm{mmol})$ were added and the reaction mixture was stirred (1 h) at $65{ }^{\circ} \mathrm{C}$. TLC analysis indicated no starting material remained. The reaction mixture was cooled and water $(20 \mathrm{~mL})$ was added to give a clear colourless solution. Following neutralization with $2 \mathrm{M} \mathrm{HCl}(\mathrm{aq})$ to $\mathrm{pH} 7$ and upon stirring, the reaction turned cloudy. Stirring was stopped and the reaction mixture placed in the fridge overnight. The precipitate was filtered, washed with water $(2 \times 5 \mathrm{~mL})$ and $\mathrm{MeOH}(2 \times 5 \mathrm{~mL})$ and then dried under high vacuum to yield compound $5(400 \mathrm{mg}, 85 \%)$ as a white chalky solid. The filtrate plus washings were evaporated under reduced pressure. Water $(10 \mathrm{~mL})$ was added to the residues and heated to boiling to give a clear solution. On cooling overnight in the fridge a small amount of solid crystallized. Work up as before gave a further crop of compound 5 (444 mg, 94\%) as light yellow fine crystals. $\mathrm{mp}>280{ }^{\circ} \mathrm{C}$. $R_{f} 0.15$ (EtOAc-MeOHconc. aq NH $3,5: 2: 2) . R_{f} 0.66$ (MeOH). IR $\left(\mathrm{cm}^{-1}\right)$ 3504, 3214, 2925, 1686, 1605, 1392, 1235, 1086, 807. ${ }^{1} \mathrm{H}$ NMR (250.1 MHz, DMSO- $d_{6}$ ): $\delta 2.38$ (ddd, J 10.0, 5.0, $\left.2.5 \mathrm{~Hz}, 1 \mathrm{H}, 2^{\prime}-\mathrm{CH} \alpha\right), 2.79\left(\mathrm{~m}, 1 \mathrm{H}, 2^{\prime}-\mathrm{CH} \beta\right), 3.63\left(\mathrm{~m}, 2 \mathrm{H}, 5^{\prime}-\mathrm{CH}_{2}\right)$, 
$3.93\left(\mathrm{~m}, 1 \mathrm{H}, 4^{\prime}-\mathrm{CH}\right), 4.47$ (br s, 1H, 3'-CH), 5.03 (br s, 1H, 5'-OH), 5.35 (br s, 1H, 3'-OH), 6.57 (t, 1H, J $7.5 \mathrm{~Hz}, 1^{\prime}-$ $\mathrm{CH}), 8.29(\mathrm{~s}, 1 \mathrm{H}, 2-\mathrm{H}), 8.73(\mathrm{~s}, 1 \mathrm{H}, 6-\mathrm{CH}), 8.91,(\mathrm{~s}, 1 \mathrm{H}, 9-\mathrm{CH}), 12.30(\mathrm{v} \mathrm{br}, 1 \mathrm{H}, \mathrm{NH}) .{ }^{13} \mathrm{C} \mathrm{NMR}(62.9 \mathrm{MHz}, \mathrm{DMSO}-$ $\left.d_{6}\right): \delta 39.31\left(2^{\prime}-\mathrm{CH}_{2}\right), 61.65\left(5^{\prime}-\mathrm{CH}_{2}\right), 70.76\left(4^{\prime}-\mathrm{CH}\right), 83.69\left(3^{\prime}-\mathrm{CH}\right), 87.99\left(1^{\prime}-\mathrm{CH}\right), 114.30(\mathrm{C}), 125.94(\mathrm{CH}), 134.71$ (C), 147.45 (CH), 147.51 (CH), 150.19 (C), 154.86 (C), 162.01 (CO). MS (FAB): $m / z$ (\%) $304\left(\mathrm{M}^{+}+\mathrm{H}, 11\right), 216$ (16), 119 (43), 114 (22), 87 (41), 82 (100). HRMS (Cl): $m / z\left[\mathrm{M}^{+}+\mathrm{Na}\right]$ calcd for $\mathrm{C}_{13} \mathrm{H}_{13} \mathrm{~N}_{5} \mathrm{O}_{4} \mathrm{Na}$; 326.0865. Found: 326.0870.

5'-O-(4.4'-Dimethoxytrityl)-3-(2'-deoxy- $\beta$-D-ribofuranosyl)-8H-imidazo[4',5':5,6]pyrido[2,3- $d$ ]pyrimidin-8-one (28). After co-evaporation with dry pyridine $(2 \times 15 \mathrm{~mL})$, compound 5 (0.36 g, $1.2 \mathrm{mmol})$ was mixed with dry pyridine $(20 \mathrm{~mL})$ and DIPEA $(0.5 \mathrm{~mL}, 40 \mathrm{mmol})$ under argon. DMTrCl $(2.4 \mathrm{~g}, 7.1 \mathrm{mmol})$ was added and the reaction mixture stirred at $\mathrm{rt}$. After $1 \mathrm{~h}$ all the compound $\mathbf{5}$ had dissolved to give a clear yellow solution. TLC analysis indicated negligible starting material and three significant fluorescent spots that stained brown with vanillin reagent. The reaction mixture was quenched with $\mathrm{MeOH}(5 \mathrm{~mL})$ and evaporated under high vacuum using a water bath temperature of $40{ }^{\circ} \mathrm{C}$ to give a viscous orange oil. TLC analysis indicated that the fluorescent spot of highest $R_{f}$ value observed prior to evaporation had degraded and two fluorescent spots were observed, the compound with lower $R_{f}$ value being the major product. Following storage overnight at $-20{ }^{\circ} \mathrm{C}$, the reaction mixture was separated by dry column vacuum chromatography using gradient elution $\left(5 \% 2 \mathrm{M} \mathrm{NH_{3 } -}\right.$ $\mathrm{MeOH}$ in EtOAc to $15 \% 2 \mathrm{M} \mathrm{NH}_{3}-\mathrm{MeOH}$ in EtOAc) to yield compound $\mathbf{2 8}$ and a slower running minor compound that was identified as the ditritylated derivative of compound 28. The column was further eluted with EtOAc$\mathrm{MeOH}$-conc. aq $\mathrm{NH}_{3}(10: 1: 1)$ to give a small amount of recovered starting material $\mathbf{5}$ with a further small fraction of compound $\mathbf{2 8}$ that was combined with the previous fractions to give compound $28(0.44 \mathrm{~g}, 61 \%)$ as a colourless solid. $\mathrm{mp}$ (decomp.) $>150{ }^{\circ} \mathrm{C}$. $R_{f} 0.28$ (EtOAc-MeOH-conc. aq NH$\left., 10: 1: 1\right) .{ }^{1} \mathrm{H} \mathrm{NMR}(250.1 \mathrm{MHz}$, DMSO-d $\left.d_{6}\right): \delta 2.35\left(\mathrm{~m}, 1 \mathrm{H}, 2^{\prime}-\mathrm{CH}\right), 2.92\left(\mathrm{~m}, 1 \mathrm{H}, 2^{\prime}-\mathrm{CH}\right), 3.25\left(\mathrm{~m}, 2 \mathrm{H}, 5^{\prime}-\mathrm{CH}_{2}\right), 3.35\left(\mathrm{~s}, 3 \mathrm{H}, \mathrm{OCH}_{3}\right), 3.40(\mathrm{~s}, 3 \mathrm{H}$, $\left.\mathrm{OCH}_{3}\right), 4.03\left(\mathrm{~m}, 1 \mathrm{H}, 4^{\prime}-\mathrm{CH}\right), 4.52\left(\mathrm{br} \mathrm{s}, 1 \mathrm{H}, 3^{\prime}-\mathrm{CH}\right), 5.39$ (d, J $\left.5.0 \mathrm{~Hz}, 1 \mathrm{H}, 3^{\prime}-\mathrm{OH}\right), 6.57\left(\mathrm{t}, 1 \mathrm{H}, J 6.7 \mathrm{~Hz}, 1^{\prime}-\mathrm{CH}\right), 6.63-$ $7.27(\mathrm{~m}, 13 \mathrm{H}, \mathrm{Ar}), 8.30(\mathrm{~s}, 1 \mathrm{H}, 2-\mathrm{H}), 8.70(\mathrm{~s}, 1 \mathrm{H}, 6-\mathrm{CH}), 8.80,(\mathrm{~s}, 1 \mathrm{H}, 9-\mathrm{CH}), 12.50(\mathrm{br}, 1 \mathrm{H}, \mathrm{NH}) .{ }^{13} \mathrm{C} \mathrm{NMR}(250.1$ $\left.\mathrm{MHz}, \mathrm{DMSO}-d_{6}\right): \delta 38.50\left(2^{\prime}-\mathrm{CH}_{2}\right), 54.93\left(\mathrm{OCH}_{3}\right), 54.93\left(\mathrm{OCH}_{3}\right), 64.50\left(5^{\prime}-\mathrm{CH}_{2}\right), 70.77\left(4^{\prime}-\mathrm{CH}\right), 83.50\left(3^{\prime}-\mathrm{CH}\right)$, $85.38\left(\mathrm{CAr}_{3}\right), 86.50\left(1^{\prime}-\mathrm{CH}\right), 112.9(\mathrm{CH}), 113.0(\mathrm{CH}), 114.50(\mathrm{C}), 126.30(\mathrm{CH}), 126.53(\mathrm{CH}), 127.64(\mathrm{CH}), 129.59$ (C), 129.80 (C), $134.90(\mathrm{CH}), 135.52$ (C), $135.62(\mathrm{C}), 144.97(\mathrm{CH}), 146.50(\mathrm{CH}), 147.50(\mathrm{CH}), 149.95(\mathrm{CH}), 157.9$ (C-OMe), 158.00 (C-OMe), 162.50 (CO). HRMS (CI) $[\mathrm{M}+\mathrm{Na}]^{+}$calcd for $\mathrm{C}_{34} \mathrm{H}_{31} \mathrm{~N}_{5} \mathrm{O}_{6} \mathrm{Na}$; 628.2172. Found: 628.2178. Analysis of the ditritylated derivative $\left(\mathrm{R}^{1}=\mathrm{R}^{2}=\mathrm{DMTr}\right)$ of compound 28: $\mathrm{HRMS}(\mathrm{Cl})\left[\mathrm{M}^{+}+\mathrm{Na}\right] \mathrm{calcd}$ for $\mathrm{C}_{55} \mathrm{H}_{49} \mathrm{~N}_{5} \mathrm{O}_{8} \mathrm{Na}$ 930.3479. Found: 930.3468.

\section{5'-O-(4,4'-Dimethoxytrityl)-3-(2'-deoxy- $\beta$-D-ribofuranosyl)-8H-imidazo[4',5':5,6]pyrido[2,3-d]pyrimidin-8-} one $\mathbf{3}^{\prime}$-O-(2-cyanoethyl)- $\mathbf{N}, \mathbf{N}$-(diisopropyl)-phosphoramidite (30). DIPEA $(0.18 \mathrm{~g}, 1.8 \mathrm{mmol}$ ) was added to a solution of compound $28(0.34 \mathrm{~g}, 0.56 \mathrm{mmol})$ in dry THF under argon. 2-Cyanoethyl- $N, N$ diisopropylchlorophosphoramidite $29(0.18 \mathrm{~g}, 0.76 \mathrm{mmol})$ was added and the reaction mixture was stirred at rt ( $3 \mathrm{~h}$ ). A precipitate formed and TLC analysis indicated negligible starting material 28 and a new product spot. The reaction mixture was evaporated under reduced pressure and stored over the weekend at $-20{ }^{\circ} \mathrm{C}$. The reaction mixture was separated by dry column vacuum chromatography using gradient elution $\left(5 \% 2 \mathrm{M} \mathrm{NH}_{3}-\right.$ $\mathrm{MeOH}$ in EtOAc to $15 \% 2 \mathrm{M} \mathrm{NH}-\mathrm{MeOH}$ in EtOAc) to yield the crude title compound 30 (0.34 g, $75 \%$ ). Purification by repeated ( $x$ 7) precipitation of a solution of compound 30 in $\mathrm{CH}_{2} \mathrm{Cl}_{2}$ ( $3 \mathrm{~mL}$ ) using petroleum ether (bp $60-80{ }^{\circ} \mathrm{C}, 300 \mathrm{~mL}$ ) gave the pure compound $30(0.23 \mathrm{~g}, 51 \%)$ as a white powder. $R_{f} 0.40$ (EtOAc-2M $\mathrm{NH}_{3}$ in $\left.\mathrm{MeOH}, 10: 1\right) .{ }^{1} \mathrm{H}$ NMR $\left(250.1 \mathrm{MHz}, \mathrm{DMSO}-d_{6}\right): \delta\left(\mathrm{m}, 12 \mathrm{H}, 4 \times \mathrm{CH}_{3}\right), 2.67$ and $2.77(\mathrm{dt}, 2 \mathrm{H}, \mathrm{J} 26.5,5.8 \mathrm{~Hz}$, $\left.\mathrm{CH}_{2} \mathrm{OP}\right), 2.88\left(\mathrm{t}, 2 \mathrm{H}, \mathrm{J} 5.9 \mathrm{~Hz}, \mathrm{CH}_{2} \mathrm{CN}\right), 3.11\left(\mathrm{~m}, 2 \mathrm{H}, 2^{\prime}-\mathrm{CH}\right), 3.18-3.80(\mathrm{~m}, 2 \mathrm{H}, 2 \times \mathrm{NCH}), 3.65(2 \times \mathrm{s}, 6 \mathrm{H}, 2 \times$ $\left.\mathrm{CH}_{3} \mathrm{O}\right), 4.05\left(\mathrm{~m}, 2 \mathrm{H}, 5^{\prime}-\mathrm{CH}_{2}\right), 4.18\left(\mathrm{~m}, 1 \mathrm{H}, 4^{\prime}-\mathrm{CH}\right), 4.79\left(\mathrm{~m}, 1 \mathrm{H}, 3^{\prime}-\mathrm{CH}\right), 6.57\left(\mathrm{~m}, 1 \mathrm{H}, 1^{\prime}-\mathrm{CH}\right), 6.75-6.61(\mathrm{~m}, 4 \mathrm{H}$, Ar), 7.10-7.35 (m, $9 \mathrm{H}, \mathrm{Ar}), 8.30(\mathrm{~s}, 1 \mathrm{H}, 2-\mathrm{CH}), 8.71(\mathrm{~s}, 1 \mathrm{H}, 6-\mathrm{CH}), 8.83(\mathrm{~s}, 1 \mathrm{H}, 9-\mathrm{CH}), 12.48(\mathrm{br} \mathrm{s}, 1 \mathrm{H}, \mathrm{NH}) .{ }^{31} \mathrm{P}$ 
NMR (101.3 MHz, DMSO- $\left.d_{6}\right): \delta 147.8,148.6$. HRMS (Cl) $m / z\left[\mathrm{M}^{+}+\mathrm{Na}\right]$ calcd for $\mathrm{C}_{43} \mathrm{H}_{48} \mathrm{~N}_{7} \mathrm{O}_{7} \mathrm{Na}$ : 828.3251. Found 828.3264.

8-Amino-3-(2'-deoxy- $\beta$-D-ribofuranosyl)-imidazo[4',5':5,6]pyrido[2,3- $d]$ pyrimidine (26). A suspension of compound 24 (100 mg, $0.36 \mathrm{mmol})$ in excess diethoxymethylacetate $(2 \mathrm{~mL})$ was heated to reflux. The reaction was followed by $\mathrm{TLC}$ (silica gel, $\mathrm{CHCl}_{3}-\mathrm{MeOH}, 5: 1$ ) and after two hours, there was no starting material present. The excess reagent was removed to give a light brown oil that was redissolved in methanolic ammonia ( $25 \mathrm{~mL}$ ) and stirred overnight at $\mathrm{rt}$. The $\mathrm{MeOH}$ was removed under reduced pressure and the residue redissolved into $25 \%$ aqueous acetic acid $(25 \mathrm{~mL}$ ) and stirred overnight. Following the removal of solvent, water was added (10 $\mathrm{mL}$ ) and the acidity adjusted to $\mathrm{pH} 6$ with $1 \mathrm{M}$ aqueous $\mathrm{NaOH}$. The reaction mixture was then chilled overnight and the precipitate recrystallised from water to give compound 4 (51 $\mathrm{mg}, 47 \%$ ) as colourless, needle-like crystals; $\mathrm{mp}>280^{\circ} \mathrm{C}$. IR ( $\left.\mathrm{cm}^{-1}\right)$ : $3085,1684,1577,1507,1419,1091,916,812 .{ }^{1} \mathrm{H}$ NMR $\left(250.1 \mathrm{MHz}, \mathrm{DMSO}-d_{6}\right)$ : $\delta 2.36\left(\mathrm{~m}, 1 \mathrm{H}, 2^{\prime}-\mathrm{CH}\right), 2.83\left(\mathrm{~m}, 1 \mathrm{H}, 2^{\prime}-\mathrm{CH}\right), 3.63\left(\mathrm{~m}, 2 \mathrm{H}, 5^{\prime}-\mathrm{CH}_{2}\right), 3.93\left(\mathrm{~m}, 1 \mathrm{H}, 3^{\prime}-\mathrm{CH}\right), 4.49\left(\mathrm{~s}, 1 \mathrm{H}, 5^{\prime}-\mathrm{OH}\right), 5.15(\mathrm{t}$, $\left.1 \mathrm{H}, J 5.6 \mathrm{~Hz}, 4^{\prime}-\mathrm{CH}\right), 5.40\left(\mathrm{~d}, 1 \mathrm{H}, J 2.7 \mathrm{~Hz}, 3^{\prime}-\mathrm{OH}\right), 6.74\left(\mathrm{t}, 1 \mathrm{H}, J 6.3 \mathrm{~Hz}, 1^{\prime}-\mathrm{CH}\right), 8.09$ (br s, 2H, NH$)^{2}, 8.51(\mathrm{~s}, 1 \mathrm{H}, 2-$ $\mathrm{CH}), 8.92(1 \mathrm{H}, \mathrm{s}, 6-\mathrm{CH}), 9.07(1 \mathrm{H}, \mathrm{s}, 9-\mathrm{CH}) .{ }^{13} \mathrm{C} \mathrm{NMR}\left(62.9 \mathrm{MHz}, \mathrm{DMSO}-d_{6}\right): \delta 39.44\left(2^{\prime}-\mathrm{CH}_{2}\right), 61.95\left(5^{\prime}-\mathrm{CH}_{2}\right)$, 71.06 (4'-CH), 83.81 (3'-CH), 88.19 (1'-CH), $106.43(\mathrm{C}), 123.80(\mathrm{CH}), 134.68(\mathrm{C}), 148.25(\mathrm{CH}), 151.01(\mathrm{C}), 155.73$ (C), $157.87(\mathrm{CH}), 164.11$ (C). MS (FAB): $m / z(\%) 303\left(\mathrm{M}^{+}+\mathrm{H}, 53\right), 187$ (31), $165(30), 152$ (46), $124(36), 120(46)$, 115 (35), 107 (100), 105 (43). HRMS (FAB): $m / z\left[\mathrm{M}^{+}+\mathrm{H}\right]$ calcd for $\mathrm{C}_{13} \mathrm{H}_{14} \mathrm{~N}_{6} \mathrm{O}_{3}$ : 303.1206. Found: 303.1209.

6,8-Diamino-3-(2'-deoxy- $\beta$-D-ribofuranosyl)-imidazo[4', $\left.5^{\prime}: 5,6\right]$ pyrido[2,3-d]pyrimidine (31). Guanidine hydrochloride $(0.40 \mathrm{~g}, 4.2 \mathrm{mmol})$ was added to a solution of sodium methoxide $(0.23 \mathrm{~g}, 4.2 \mathrm{mmol})$ in absolute $\mathrm{MeOH}(30 \mathrm{~mL})$. The mixture was stirred at $20{ }^{\circ} \mathrm{C}$ for $15 \mathrm{~min}$ before compound 24 (0.10 g, $0.36 \mathrm{mmol}$ ) was added and the mixture stirred at $145^{\circ} \mathrm{C}$ for $42 \mathrm{~h}$ in a steel bomb after which time TLC analysis (EtOAc-MeOH, 10:1) showed there was no starting material. On cooling, the solid was filtered off and washed successively with water and ethanol to give compound $31(0.52 \mathrm{~g}, 45 \%)$ as a cream solid; $\mathrm{mp}>280{ }^{\circ} \mathrm{C}$. IR $\left(\mathrm{cm}^{-1}\right): 3481,3434$, $3318,3183,3110,2887,1641,1621,1470,1356,1057,803 .{ }^{1} \mathrm{H}$ NMR $\left(250.1 \mathrm{MHz}, \mathrm{DMSO}-d_{6}\right): \delta 2.23\left(\mathrm{~m}, 1 \mathrm{H}, 2^{\prime}-\right.$ $\mathrm{CH}), 2.81\left(\mathrm{~m}, 1 \mathrm{H}, 2^{\prime}-\mathrm{CH}\right), 3.61\left(\mathrm{~m}, 2 \mathrm{H}, 5^{\prime}-\mathrm{CH}_{2}\right), 3.88\left(\mathrm{~m}, 1 \mathrm{H}, 4^{\prime}-\mathrm{CH}\right), 4.44\left(\mathrm{~m}, 1 \mathrm{H}, 3^{\prime}-\mathrm{CH}\right), 5.17\left(\mathrm{t}, 1 \mathrm{H}, 5^{\prime}-\mathrm{OH}\right), 5.37$ (d, 1H, 3'-OH), 6.19 (br s, 2H, NH $), 6.42\left(\mathrm{t}, 1 \mathrm{H}, J 6.5 \mathrm{~Hz}, 1^{\prime}-\mathrm{CH}\right), 7.44$ (br s, 2H, NH $\mathrm{NH}_{2}, 8.64(\mathrm{~s}, 1 \mathrm{H}, 6-\mathrm{CH}), 8.83(1 \mathrm{H}$, s, 9-CH). ${ }^{13} \mathrm{C}$ NMR (62.9 MHz, DMSO-d $\left.d_{6}\right): \delta 39.17\left(2^{\prime}-\mathrm{CH}_{2}\right), 62.08\left(5^{\prime}-\mathrm{CH}_{2}\right), 71.17\left(4^{\prime}-\mathrm{CH}\right), 83.84\left(3^{\prime}-\mathrm{CH}\right), 88.01\left(1^{\prime}-\right.$ $\mathrm{CH}), 102.19(\mathrm{C}), 123.83(\mathrm{CH}), 131.33(\mathrm{C}), 145.35(\mathrm{CH}), 150.76(\mathrm{C}), 158.27(\mathrm{CH}), 162.78(\mathrm{C}), 164.21$ (C). MS (FAB): $m / z$ (\%) $318\left(\mathrm{M}^{+}+\mathrm{H}, 91\right), 202$ (100), 141 (15), 121 (9). HRMS (FAB) $m / z\left[\mathrm{M}^{+}+\mathrm{Na}\right.$ ] calcd. for $\mathrm{C}_{13} \mathrm{H}_{15} \mathrm{~N}_{7} \mathrm{NaO}_{3}$ : 340.1134. Found: 340.1129 .

\section{Acknowledgements}

We thank Scotia Pharmaceuticals for a studentship (to RC), the Royal Society for a grant (to WF), and the EPSRC Mass Spectrometry Centre for high-resolution mass spectra.

\section{Supplementary Material}

Copies of ${ }^{1} \mathrm{H}$ and ${ }^{13} \mathrm{C}$ NMR spectra are provided as supplementary material. 


\section{References}

1. Leonard, N. J.; Sprecker, M. A; Morrice, G. A. J. Am. Chem. Soc. 1976, 98, 3987-3994. https://doi.org/10.1021/ja00429a040

2. Scopes, D. I. C; Barrio, J. R.; Leonard, N. J. Science 1977, 195, 296-298.

https://doi.org/10.1126/science.188137

3. Leonard, N. J.; Keyser, G. E. Proc. Natl. Acad. Sci. U.S.A. 1979, 76, 4262-4264.

https://doi.org/10.1073/pnas.76.9.4262

4. Leonard, N. J.; Acc. Chem. Res. 1982, 15, 128-135.

https://doi.org/10.1021/ar00077a001

5. Lessor, R. A.; Gibson, K. J.; Leonard, N. J. Biochemistry 1984, 23, 3868-3873.

https://doi.org/10.1021/bi00312a012

6. Liu, H.; Gao, J.; Maynard, L.; Saito Y. D.; Kool, E. T. J. Am. Chem. Soc. 2004, 126, 1102-1109.

https://doi.org/10.1021/ja038384r

7. Gao, J.; Liu, H.; Kool, E. T. Angew. Chem. Int. Ed. 2005, 44, 3118-3122.

https://doi.org/10.1002/anie.200500069

8. Lynch, S. R.; Liu, H.; Gao, J.; Kool, E. T. J. Am. Chem. Soc. 2006, 128, 14704-14711.

https://doi.org/10.1021/ja065606n

9. Lee, A. H. F.; Kool, E. T. J. Org. Chem. 2005, 70, 132-140.

https://doi.org/10.1021/jo0483973

10. Lee, A. H. F.; Kool, E. T. J. Am. Chem. Soc. 2006, 128, 9219-9230.

https://doi.org/10.1021/ja0619004

11. Krueger, A. T.; Lu, H.; Lee, A. H. F.; Kool, E. T. Acc. Chem. Res. 2007, 40, 141-150.

https://doi.org/10.1021/ar068200o

12. Delaney, J. C.; Gao, J.; Lui, H.; Shrivastav, N.; Essigmann, J. M.; Kool, E. T. Angew. Chem. Int. Ed. Engl. 2009, 48, 4524-4527.

https://doi.org/10.1002/anie.200805683

13. Krueger, A. T.; Peterson, L. W.; Chelliserry, J.; Kleinbaum, D. J.; Kool, E. T. J. Am. Chem. Soc. 2011, 133, 18447-18451.

https://doi.org/10.1021/ja208025e

14. Furukawa, K.; Hattori, M.; Ohki, T.; Kitamura, Y.; Kitade, Y.; Ueno, Y. Bioorg. Med. Chem. 2012, 20, 16-24. https://doi.org/10.1016/i.bmc.2011.11.045

15. Hattori, M.; Ohki, T.; Yanase, E.; Ueno, Y. Bioorg. Med. Chem. Lett. 2012, 22, 253-257. https://doi.org/10.1016/j.bmcl.2011.11.022

16. Hayai, A.; Maeda, Y.; Ueno, Y. Bioorg. Med. Chem. Lett. 2016, 26, 3552-3555.

https://doi.org/10.1016/i.bmcl.2016.06.024

17. Humphries, M. J.; Ramsden, C. A. Synthesis 1999, 985-992.

https://doi.org/10.1055/s-1999-3500

18. Clayton, R.; Davis, M. L.; Fraser, W.; Li, W.; Ramsden, C. A. Synlett 2002, 1483-1486.

https://doi.org/10.1055/s-2002-33505

19. Crawford, J. A.; Fraser, W.; Ramsden, C. A. Synthesis 2009, 1271-1278.

https://doi.org/10.1055/s-0028-10880

20. Fraser, W. Adv. Heterocycl. Chem. 2012, 107, 1-39.

https://doi.org/10.1016/B978-0-12-396532-5.00001-9 
21. Hoffer, M. Chem. Ber. 1960, 93, 2777-2781.

https://doi.org/10.1002/cber.19600931204

22. Chin, T.-M.; Huang, L. K.; Kan, L. J. Chin. Chem. Soc. 1997, 44, 413-416.

https://doi.org/10.1002/jccs.199700062

23. Rolland, V.; Kotera, M.; Lhomme, J.; Synth. Commun. 1997, 27, 3505-3511.

https://doi.org/10.1080/00397919708007071

24. Dhimitruka, I.; SantaLucia Jr., J. Synlett, 2004, 335-337.

25. Bergstrom, D. E.; Zhang, P.; Travis Johnson, W. Nucleic Acids Res. 1997, 25, 1935-1942.

https://doi.org/10.1093/nar/25.10.1935

26. Hubbard, A. J.; Jones, A. S.; Walker, R. T. Nucleic Acids Res. 1984, 12, 6827-6837.

https://doi.org/10.1093/nar/12.17.6827

27. Štimac, A.; Kobe, J. Carbohydr. Res. 2000, 329, 317-324.

https://doi.org/10.1016/S0008-6215(00)00186-5

28. McKillop, A; Wright, D. E.; Podmore, M. L.; Chambers, R. K. Tetrahedron 1983, 39, 3797-3800. https://doi.org/10.1016/S0040-4020(01)88621-X

29. Al-Shaar, A. H. M.; Gilmour, D. W.; Lythgoe, D. J.; McClenaghan, I.; Ramsden, C. A. J. Chem. Soc. Perkin Trans. 1, 1992, 2779-2788.

https://doi.org/10.1039/p19920002779

30. Al-Shaar, A. H. M.; Chambers, R. K.; Gilmour, D. W.; Lythgoe, D. J.; McClenaghan, I.; Ramsden, C. A. J. Chem. Soc. Perkin Trans. 1, 1992, 2789-2811.

https://doi.org/10.1039/P19920002789

31. Harris, P. A; Pendergast, W. J. Heterocyclic Chem. 1996, 33, 319-322.

https://doi.org/10.1002/jhet.5570330218

32. Yamada, H.; Okamoto, T. Chem. Pharm. Bull. 1972, 20, 623-624.

https://doi.org/10.1248/cpb.20.623

33. Saladino, R.; Crestini, C.; Castanzo, G.; Negri, R.; Di Mauro, E. Bioorg. Med. Chem. Lett. 2001, 9, 12491253.

https://doi.org/10.1016/S0968-0896(00)00340-0

34. Marfut, J.; Parel, S. P.; Leumann, C. J. Nucleic Acids Res. 1997, 25, 1875-1882.

https://doi.org/10.1093/nar/25.10.1875

35. Groebke, K.; Hunziker, J.; Fraser, W.; Peng, L.; Diederichsen, U.; Zimmermann, K.; Holzner, A.; Leumann, C.; Eschenmoser, A. Helv. Chim. Acta 1998, 81, 375-474.

https://doi.org/10.1002/hlca.19980810302

36. Still, W. C.; Kahn, M.; Mitra, A. J. Org. Chem. 1978, 43, 2923-2925.

https://doi.org/10.1021/jo00408a041

37. Pedersen, D. S.; Rosenbohm, C. Synthesis 2001, 2431-2434.

https://doi.org/10.1055/s-2001-18722 